\title{
Integrated resource for functional and structural connectivity of the marmoset brain
}

\author{
Xiaoguang Tian ${ }^{1, *}$, Yuyan Chen², Piotr Majka ${ }^{3,7}$, Diego Szczupak¹, Yonatan Sanz \\ Perl $^{4,11}$, Cecil Chern-Chyi Yen ${ }^{5}$, Chuanjun Tong ${ }^{2}$, Kun Song ${ }^{2}$, Haiteng Jiang ${ }^{12,13}$, Daniel \\ Glen ${ }^{6}$, Gustavo Deco ${ }^{4,8,9,10}$, Marcello G. P. Rosa ${ }^{7,}{ }^{*}$, Afonso C. Silva ${ }^{1, *}$, Zhifeng \\ Liang $2,14,15,{ }^{*}$, Cirong Liu ${ }^{2,14,15, *}$
}

${ }^{1}$ Department of Neurobiology, University of Pittsburgh Brain Institute, University of Pittsburgh, Pittsburgh PA 15261, USA

${ }^{2}$ Center for Excellence in Brain Science and Intelligence Technology, Institute of Neuroscience, CAS Key Laboratory of Primate Neurobiology, Chinese Academy of Sciences, Shanghai, China

3 Laboratory of Neuroinformatics, Nencki Institute of Experimental Biology of the Polish Academy of Sciences, 02-093 Warsaw, Poland

${ }^{4}$ Center for Brain and Cognition, Computational Neuroscience Group, Department of Information and Communication Technologies, Universitat Pompeu Fabra, Roc Boronat 138, Barcelona, 08018, Spain

${ }^{5}$ Cerebral Microcirculation Section, Laboratory of Functional and Molecular Imaging, National Institute of Neurological Disorders and Stroke, National Institutes of Health (NINDS/NIH), Bethesda MD 20892, USA

${ }^{6}$ Scientific and Statistical Computing Core, National Institute of Mental Health, National Institutes of Health (NIMH/NIH), Bethesda MD 20892, USA

${ }^{7}$ Department of Physiology and Neuroscience Program, Biomedicine Discovery Institute, Monash University, Clayton, VIC 3800, Australia

8 Institució Catalana de la Recerca i Estudis Avançats (ICREA), Passeig Lluís Companys 23, Barcelona, 08010, Spain

${ }^{9}$ Department of Neuropsychology, Max Planck Institute for Human Cognitive and Brain Sciences, 04103 Leipzig, Germany

${ }^{10}$ School of Psychological Sciences, Monash University, Melbourne, Clayton VIC 3800, Australia

11 Universidad de San Andrés, Vito Dumas 284 (B1644BID), Buenos Aires, Argentina

12 Department of Neurobiology, Affiliated Mental Health Center \& Hangzhou Seventh People's Hospital, Zhejiang University School of Medicine

13 MOE Frontier Science Center for Brain Science and Brain-machine Integration, Zhejiang University, Hangzhou, China.

14 University of Chinese Academy of Sciences, Beijing, China

${ }^{15}$ Shanghai Center for Brain Science and Brain-Inspired Intelligence Technology, Shanghai, China

* Corresponding authors 


\section{Abstract}

The comprehensive integration of structural and functional connectivity data is required for the accurate modeling of brain functions. While resources for studying structural connectivity of non-human primate marmoset brains already exist, their integration with functional connectivity data has remained unavailable. Therefore, we present a comprehensive resource for marmoset brain mapping, which integrates the largest awake resting-state fMRI dataset to date (39 marmosets, 709 runs, and 12053 mins), cellularlevel neuronal-tracing dataset (52 marmosets and 143 injections), and multi-resolution diffusion MRI dataset. The combination of these data into the same MRI space allowed us to 1). map the fine-detailed functional networks and cortical parcellations; 2 ). develop a deep-learning-based parcellation generator to preserve the topographical organization of functional connectivity and reflect individual variabilities; 3 ). investigate the structural basis underlying functional connectivity by computational modeling. Our resource will broadly model the marmoset brain architecture and facilitate future comparative and translational studies of primate brains.

Keywords: Marmoset, Resting-state fMRI, Brain Networks, Parcellation, Computational model, Neuronal Tracing, Functional Connectivity, Structural Connectivity, Deep Neural Network, Non-human Primate 


\section{Introduction}

Mapping brain architecture is critical for decoding brain functions and understanding the mechanisms of different brain diseases (Buffalo et al., 2019). Non-human primate (NHP) neuroimaging provides a granular view of the evolution of the brain connectome (Thiebaut de Schotten et al., 2019) and overcomes constraints of human neuroimaging by accessing "ground truth" data from neuronal tracing (Buckner and Margulies, 2019). As one of the smallest NHP with many practical advantages and potentials in biomedical research, the common marmoset (Callithrix jacchus) has drawn considerable interest for NHP neuroimaging research (Eliades and Miller, 2017; Homman-Ludiye and Bourne, 2017; Matsuzaki and Ebina, 2020; Mitchell and Leopold, 2015; Okano, 2021; Okano and Mitra, 2015; Schultz-Darken et al., 2016; Walker et al., 2017). Via Marmoset Brain Mapping (marmosetbrainmapping.org) and the Marmoset Brain Connectivity Atlas (marmosetbrain.org), we have contributed the ultra-high-resolution ex-vivo diffusion MRI data (Liu et al., 2020), mesoscale neural tracing data (Majka et al., 2020), and structural atlases (Liu et al., 2018; Liu et al., 2021; Majka et al., 2021), which have enabled an unprecedented level of precision in analyses of the marmoset brain neuroanatomy. However, an essential component for a thorough understanding of brain architecture has been missing: the functional connectome.

Resting-state fMRI (rs-fMRI) is one of the few non-invasive imaging techniques capable of mapping the whole-brain functional connectome (Smith et al., 2013). However, existing NHP rs-fMRI data repositories have limitations. First, data-sharing initiatives of NHP neuroimaging are still at an early stage, with existing open datasets of rs-fMRI data originating in different laboratories and collected for different purposes (Milham et al., 2018). Thus, this leads to inconsistent imaging protocols and data quality, which can diminish the interpretability of meta-analyses across datasets. Second, most rs-fMRI datasets were acquired from anesthetized animals, resulting in difficulties for crossspecies studies, particularly relative to awake human brains (Hudetz, 2012). The final barrier is the practical difficulty of training animals to be fully awake during MRI scans (Milham et al., 2021; Milham et al., 2020). Given that, a platform for international 
collaborative research (PRIMatE RESOURCE EXCHANGE) was initiated to promote open resource exchange and standards for NHP neuroimaging (Messinger et al., 2021; Milham et al., 2018).

To address these challenges and limitations, in complete alignment with a strategic plan developed by the NHP imaging community (Milham et al., 2021), we developed standardized awake imaging protocols for marmosets, acquiring the most sizeable to date open-access datasets of awake rs-fMRI across two institutions, the National Institutes of Health (NIH), USA, and the Institute of Neuroscience (ION), China. Furthermore, we also integrated neuronal-tracing and different diffusion MRI datasets into the same MRI space, resulting in the comprehensive resource, which allows exploration of the relationships between structural and functional connectome. The marmoset brain mapping resource reported in this paper (available via www.marmosetbrainmapping.org) is characterized by:

1) Publicly available standardized dataset: The largest awake resting-state test-retest fMRI data available to date involve 39 adult marmosets with 709 runs (17 min per run; 12053 mins in total). All rs-fMRI data follows a standardized imaging protocol to ensure consistent data quality and interpretability.

2) Functional brain mapping: We created a comprehensive mapping of resting-state brain networks and a fine-grained cortical parcellation based on brain functional connectivity. Furthermore, we developed a deep-learning-based method to map the functional cortical parcellation onto individual brains accurately.

3) Relationship with structural connectivity: We sampled the extensive collection of NHP neuronal tracing data (52 marmosets and 143 injections) onto the same MRI space at the voxel or vertex level and integrated it with the functional MRI data mentioned above. In addition, further enhancing the capacity of our resource, we integrated extra high-resolution ex-vivo diffusion MRI and in-vivo diffusion MRI data obtained at 25 marmosets from the same cohort. On this basis, we investigated the 
relationship between structural and functional connectivity using a whole-brain computational model.

\section{Results}

\section{Highly consistent datasets}

Following the same protocols for animal training and MRI imaging, including the designs of the radiofrequency coil and MRI pulse sequences, we acquired an extensive awake resting-state $\mathrm{fMRI}$ dataset to date from 39 marmosets of two research institutes (13 from ION, age 3+/-1 years old; 26 from NIH, age 4+/-2 years old; see Supplementary Table 1 for details). This is also the same range of ages used in our previous studies of structural connectivity (Liu et al., 2020; Majka et al., 2020). For test-retest evaluation, we scanned multiple runs (17 mins/run) for each marmoset, resulting in an essentially similar data quantity of two institutes (351 ION runs and 358 NIH runs), and included two "flagship" marmosets with many runs (64 runs from the ION and 40 runs from the NIH). Besides similar quantity, the data quality of the two sites is also highly consistent, guaranteeing interpretability across datasets (Supplementary Fig. S1, see method for the details).

\section{Mapping functional brain networks}

Identifying functional networks of areas showing highly correlated fMRI signals is a key to characterizing the brain architecture. Using the independent component analysis (ICA), a data-driven approach for separating independent patterns in multivariate data, we identified 19 distinct functional networks from awake resting-state fMRI data, including 4 subcortical and 15 cortical networks (Fig. 1 and Supplementary Fig. S2). The subcortical networks included the brain stem, the thalamus, the striatum, and the cerebellum (Supplementary Fig. S2 P-S). 
The details of the 15 cortical networks were as follows. Six functional networks were characterized by the short-range connectivity, including the ventral somatomotor (Fig. 1A), the dorsal somatomotor (Fig. 1B), the premotor (Fig. 1C), the frontopolar (Fig. 1D), the orbitofrontal (Fig. 1E), and the parahippocampal/ temporopolar cortex (Fig. 1F). Two components are the auditory and salience-related networks, the first being primarily located in the auditory and insular cortices and weakly coupled with the anterior cingulate cortex (Fig. 1G), and the second (Fig. 1H) encompassing the anterior cingulate cortex. In addition, we also identified two trans-modal networks (Fig. 1I-J), including association areas in the dorsolateral prefrontal cortex (dIPFC), rostral premotor cortex, lateral and medial parietal cortices, and temporal cortex. According to a previous study (Liu et al., 2019), one is most likely the frontoparietal-like network (Fig. 1I), and the other is the default-model-like network (DMN, Fig. 1J). Importantly, the putative frontoparietal-like network has not been recognized in previous studies (Belcher et al., 2013; Ghahremani et al., 2017); The remaining five networks represent the first complete mapping of visualrelated functional networks of the marmoset cortex (Fig. 1K-O). Three networks included the primary visual cortex and parts of extrastriate areas related to far peripheral vision (Fig. 1K), near-peripheral vision (Fig. 1L), and the foveal vision (Fig. 1M). The other two networks involve hierarchically higher visual areas (Fig. $1 \mathrm{~N}-\mathrm{O}$ ), such as V3, V4, the inferior temporal cortex, the adjacent polysensory temporal cortex, and visual-related frontal regions.

Based on their spatial overlap patterns and connectivity strengths (normalized Z-scores), we combined the 15 cortical networks into network-parcellation maps (Fig. 1P-Q). Due to local connectivity being stronger than long-range connectivity, the primary map (Fig. 1P$\mathrm{Q}$, top rows) is dominated by the short-range networks (i.e., Fig. 1G, I, J, K, L, I, and O). Thus, we created the second one (Fig. 1P-Q, bottom rows) to cover the long-range connectivity that was not captured by the primary map. In sum, the two networkparcellation maps characterized the entire cortical networks and will be widely used in functional connectivity studies of the marmoset brain. 

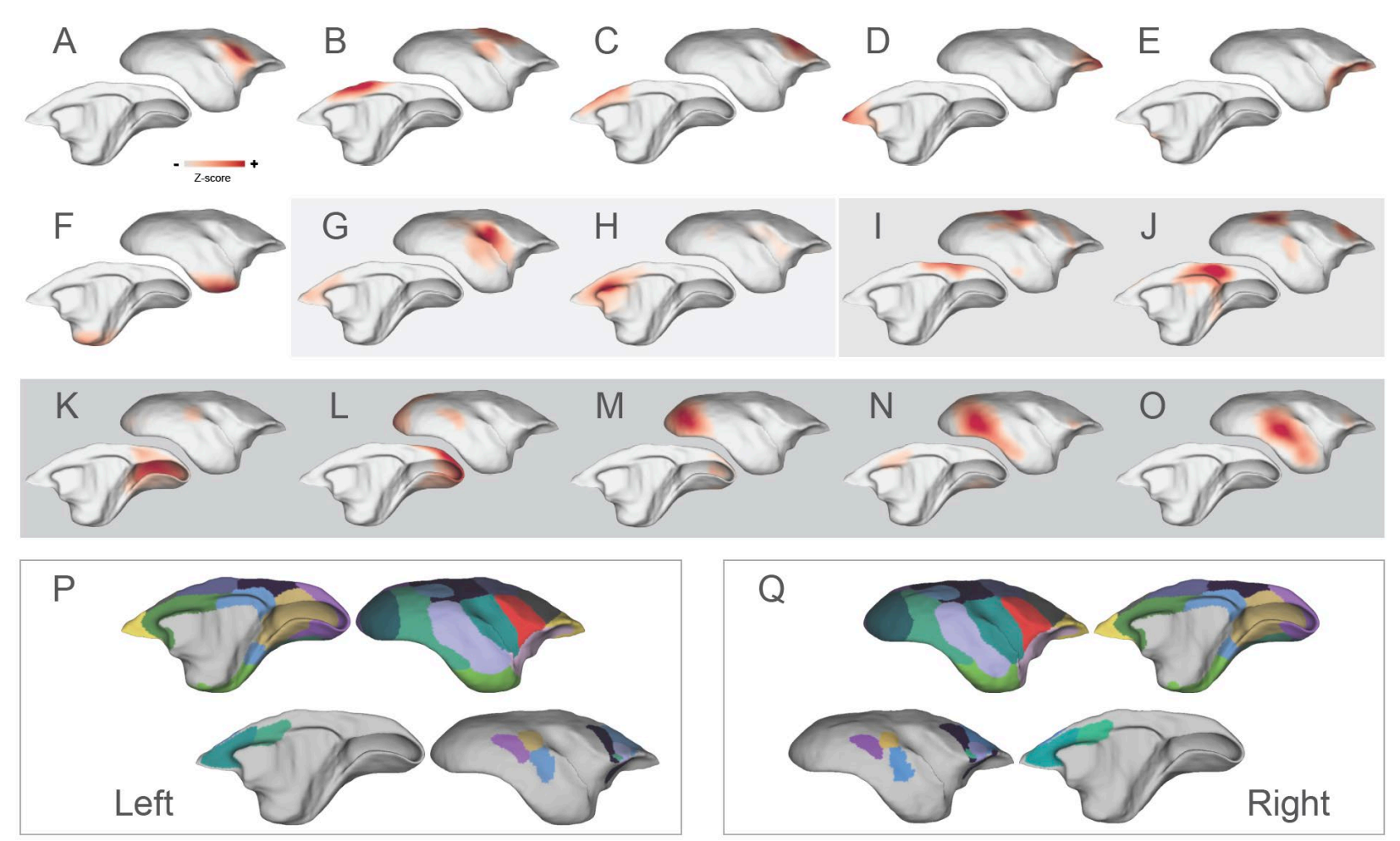

Figure 1. Identified cortical functional networks and their parcellation maps. The networks include (A) the ventral somatomotor, (B) the dorsal somatomotor, (C) the premotor, $(D)$ the frontal pole, $(E)$ the orbital frontal cortex, $(F)$ the parahippocampus and temporal pole, (G-H) the auditory and salience-related network, (I-J) two trans-modal networks, which are most-likely related to the frontoparietal network and the defaultmode-network, and $(\mathrm{K}-\mathrm{O})$ the visual-related networks from the primary visual cortex to functional higher-level regions. These networks were combined to form two networkparcellation maps $(P-Q)$, which are dominated by the networks with short-range connectivity ( $P-Q$, top rows) and with long-range connectivity ( $P-Q$, bottom rows), respectively. 


\section{Mapping functional-connectivity boundaries}

The brain network maps provided a global view of cortical functional organization. Our next aim was to characterize the cortex at a finer local scale. Here, we used the functional connectivity boundary mapping approach to identify putative borders of functional parcels (Cohen et al., 2008; Gordon et al., 2016; Wig et al., 2014), which represent an efficient way to map transitions in functional connectivity.

Population boundary maps based on the $\mathrm{ION}$, the $\mathrm{NIH}$, or combined datasets are visually similar, presenting clear functional-connectivity borders (Fig. 2A), and were highly reproducible with average Dice's coefficients for both hemispheres: 0.7 (ION-NIH), 0.71 (ION-Both), and 0.69 (NIH-Both), respectively (Supplementary Fig. S3). However, although consistent at the population level, boundary maps indicate variabilities across individuals (Fig. 2B), with an average Dice's coefficient of 0.3842 for both hemispheres (Fig. 2C-D), significantly lower than the population (the value of 0.7 ). We also found high across-session variability in the same individual, but more scanning runs efficiently enhanced the reproducibility (Fig. 2E). Therefore, the results suggest that both individual and across-session variability contribute to the low consistency of individual boundary maps, and the test-retest data are essential for improving the reliability of maps. 

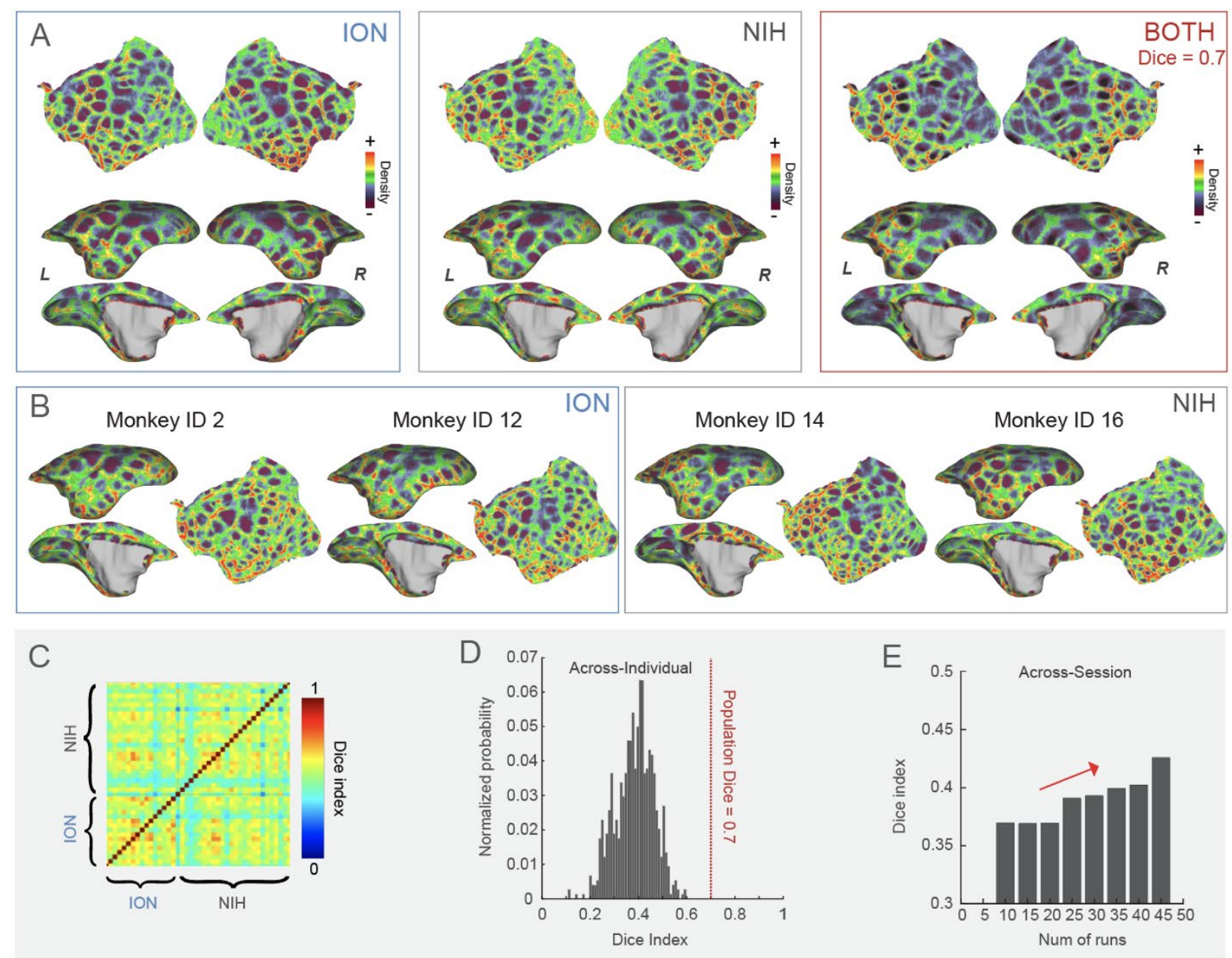

Figure 2. The functional connectivity boundary maps. (A). The population-based boundary maps from the ION, the NIH and the combined datasets. These maps are highly consistent, with the average Dice's coefficient of 0.7. (B). Boundary maps in the left hemisphere from four exemplar marmosets (two from the NIH cohort and two from the $I O N$, including the flagship marmosets). (C-D). The heatmap of the average Dice's coefficients for both hemispheres between individuals and its distribution histogram. (E). The change of the average Dice's coefficients for both hemispheres with the number of runs in the same individuals. 
Generation of functional-connectivity parcels (Marmoset Brain Mapping Atlas Version 4, MBMv4)

Because the population boundary maps are more reproducible than individual maps, we used the combined ION-NIH population boundary map to generate cortical functionalconnectivity parcels. By the detection of the local-minima, "watershed-flood" region growing, and post-optimization (Fig. 3A), we obtained 96 parcels per hemisphere (Fig. 3B). Since we processed each hemisphere independently, we compared the similarity of the parcellations of two hemispheres. The two-hemispherical parcellations are similar in the parcel sizes (Supplementary Fig. S4-A; Wilcoxon paired signed-rank test, $N=96$, $p=0.7981$ ) and functional connectivity patterns between vertices within the same parcel (Supplementary Fig. 4-B; Wilcoxon paired signed-rank test, $N=96, p=0.411$ ). This leftright symmetry corroborates the reliability of our parcel generation. For continuity with previously released resources (Liu et al., 2020; Liu et al., 2018; Liu et al., 2021), we named this functional-connectivity-based parcellation of the cortex "Marmoset Brain Mapping Atlas Version 4" (MBMv4).

To estimate the validity of the generated functional parcels, we used the distancecontrolled boundary coefficient (DCBC) (King et al., 2019). The basic idea of DCBC is that when a boundary divides two functionally homogenous regions, for any equal distance on the cortical surface, the functional connectivity pattern between vertices within the same parcel should be higher than that between vertices in different parcels (Fig. 3C). In other words, a higher DCBC (within - between) means higher within-parcel homogeneity and higher between-parcel heterogeneity. We calculated the DCBC between the vertex pairs using a range of spatial bins $(0-4 \mathrm{~mm})$ with a $0.5 \mathrm{~mm}$ step (the spatial resolution of the rs-fMRI data). Here, we compared the fit of the functional map represented by MBMv4 with existing structural cortical parcellations, including MBMv1 atlas (Liu et al., 2018), the digital reconstruction of the Paxinos et al. (2012) atlas (Liu et al., 2018; Majka et al., 2016), and the RIKEN atlas (Woodward et al., 2018). The result of $\mathrm{DCBC}$ in Fig. 3D demonstrates that MBMv4 has the best performance for the presentation of functional connectivity (the average DCBC values were $0.0186,0.0135,0.0177,0.0330$ 
for RIKEN, MBMv1, Paxinos, and MBMv4 atlas; multiple comparisons for One-Way ANOVA $\left.F_{(3,8556)}=22.44, p=1.81 \times 10^{-14}\right)$.

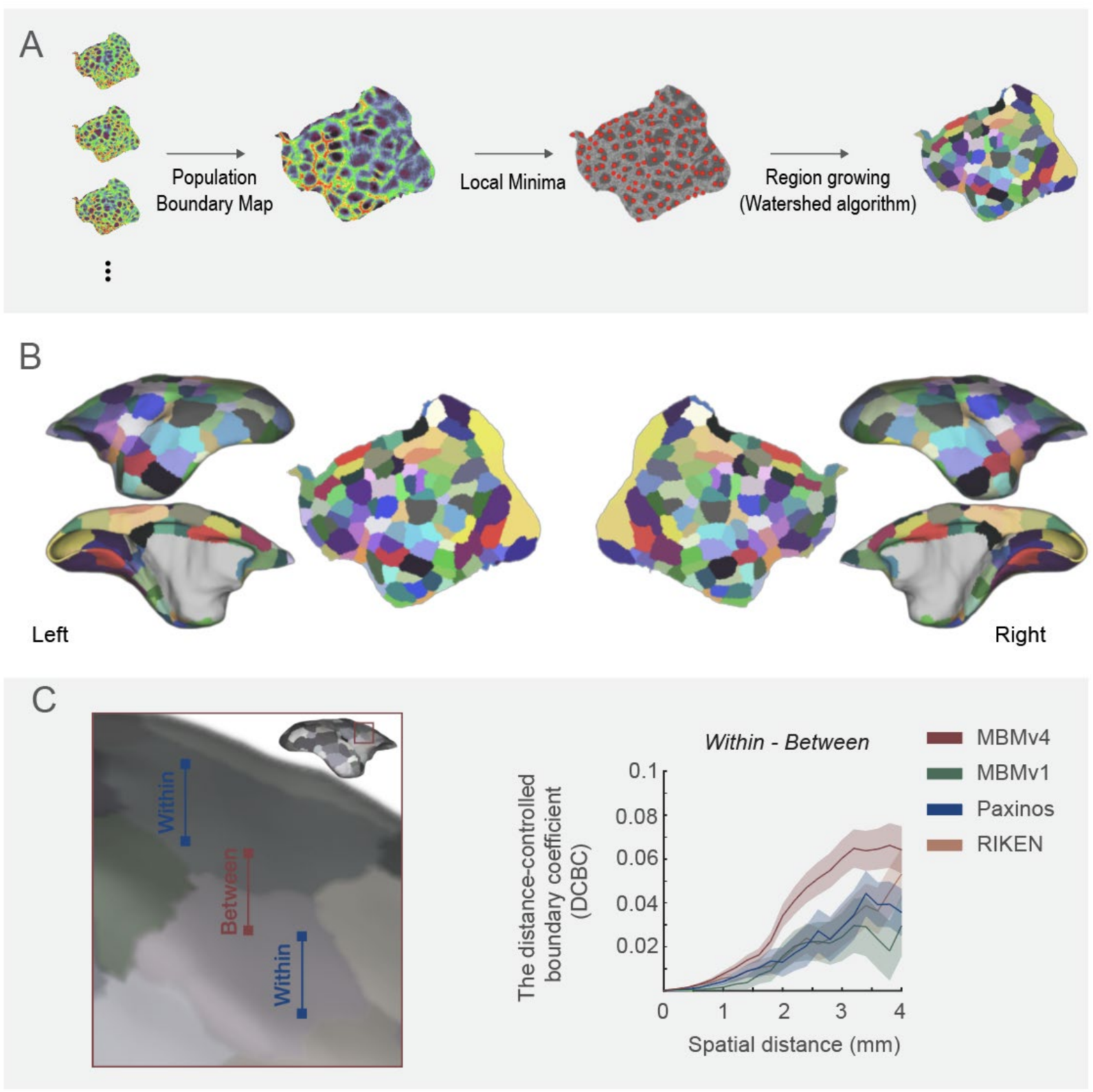

Figure 3. Marmoset Brain Mapping Atlas Version 4 (MBMv4). (A) The processing procedure includes generating the population functional-connectivity boundary maps, defining the local minima for seeding, and generating parcels by the "watershed" algorithm. (B) The resulting 96 functional-connectivity parcels per hemisphere overlaid on the white matter surface and flat map of MBMv3 (Liu et al., 2021). (C) The evaluation metric of distance-controlled boundary coefficient (DCBC). According to parcellations 
(Right panel: MBMv1, MBMv4, Paxinos, and RIKEN atlas), all pairs of voxels/vertices were categorized into "within" or "between" parcels (left panel), and the DCBC metric was calculated by the differences (within-between) in functional connectivity as the function of distance on the surface $(0-4 \mathrm{~mm}$ in steps of $0.5 \mathrm{~mm})$. Data are presented in mean $+/-$ s.e.m.

\section{Mapping MBMv4 in Individual Brains by Deep Neural Networks}

To overcome the limitation of variable individual boundary maps (Fig. 2C-E), we employed a deep-learning approach for the individual mapping from MBMv4 (Fig. 4A). First, based on the population-level whole-brain functional connectivity, we trained a deep neural network classifier for each parcel to learn the associated fingerprint of functional connectivity. Then, the trained networks distinguished the goal parcel for every marmoset based on the corresponding functional connectivity of the searching area, consisting of the goal parcel and its neighbors. Due to the overlap of searching areas, vertices could belong to multiple parcels. Therefore, we only kept these vertices attributed to a single parcel as the seeds for the regional growing by the "watershed" algorithm. This iterative region-growing procedure would assign all vertices to a parcel, resulting in an individual cortical parcellation.

Since individual parcellation should be reasonably close to the population definition (Glasser et al., 2016; Hacker et al., 2013), we compared the population-based MBMv4 parcellation and the automatically generated individual parcellations. By calculating the percentage of vertices sharing the same labels from both hemispheres (the metric of concordance), we found that the individual parcellations from all marmosets are similar to MBMv4 with an average of $90 \%$ concordance (Fig. $4 \mathrm{~B}$, the violin/box plot on the left, the examples on the right). Using the test-retest dataset, we revealed the consistency of the individual parcellations across different sessions (Fig. 4C, the violin/box plot on the left, and examples on the right). The across-session analysis yielded an average of $86.7 \%$ concordance, lower than the average value of $91.3 \%$ across-individual similarities. Furthermore, we observed that the lateral prefrontal cortex and occipital-temporal cortex 
had higher across-individual and across-session mapping variabilities (Supplementary Fig. S5), consistent with previous findings in human studies (Gordon et al., 2016; Mueller et al., 2013). Thus, the deep-learning approach efficiently adjusts the parcel borders to reflect the individual variabilities while maintaining high consistency with the population parcellation.

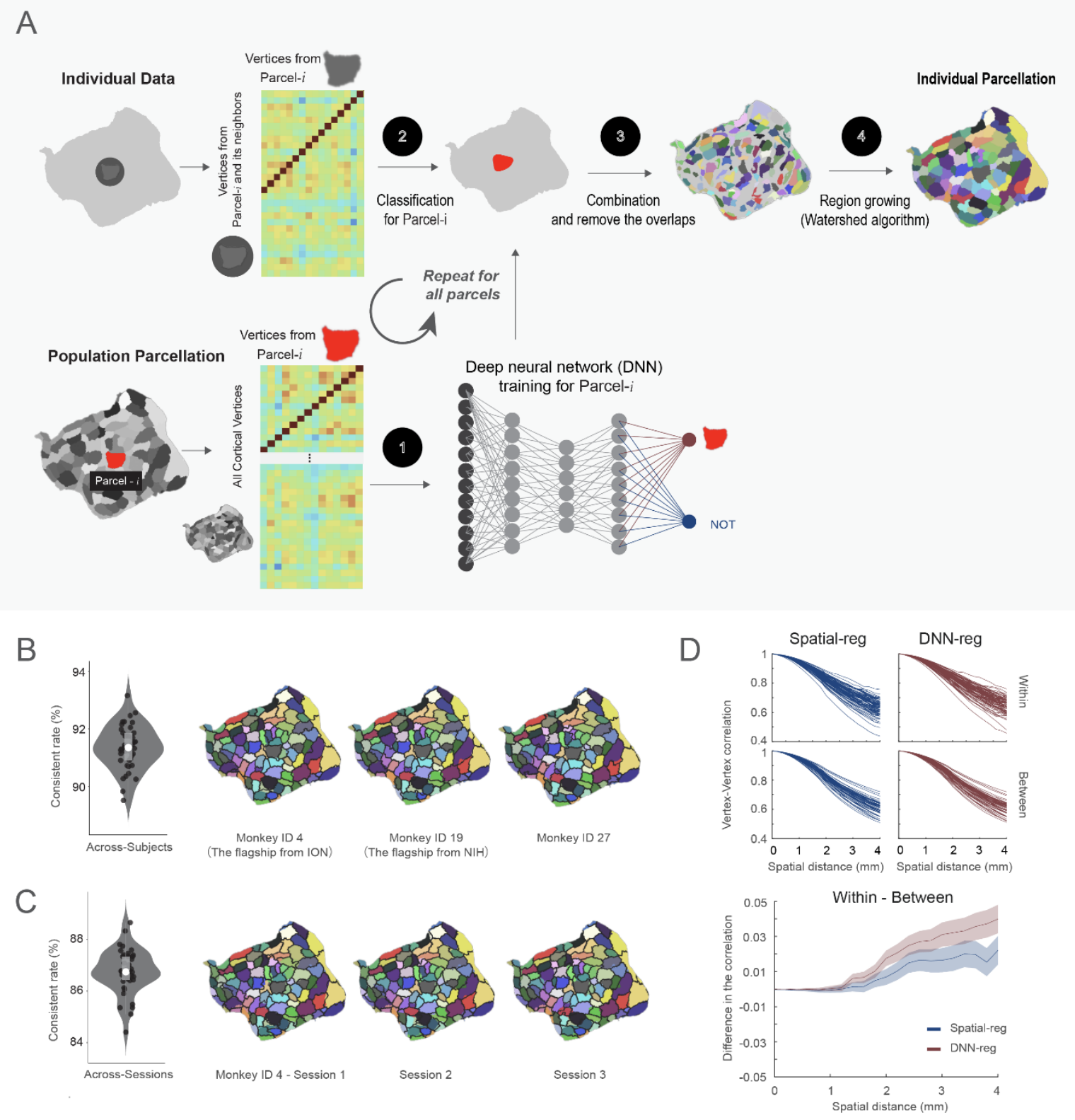

Figure 4. Mapping individual functional-connectivity parcellation. (A) An overview of individual mapping based on the deep neural network approach. (B) MBMv4 Mapping of 
each individual. Left panel: the concordance between the population MBMv4 and individual parcellations. Data are presented by the violin and the box plots (25th percentile and 75 percentile), in which the white point represents the average value; Right panel: three examples of individual parcellations. The underlay (color-coded) presents the population MBMv4, and the overlay (black border) shows the individual parcellations. (C) Mapping of MBMv4 per session. Left panel: The concordance between every individual parcellation and the corresponding parcellation using one session data; Right panel: representative parcellations of three sessions from one marmoset. The color-coded underlay represents individual parcellation, while the black border overlay shows the session-based parcellation. (D) The distance-controlled boundary coefficient (DCBC) for the individual parcellation generated by the spatial registration (Spatial-reg, blue) and the deep neural network (DNN-reg, red). Top panel: the functional connectivity for all pairs of vertices within the same parcel and between parcels for DNN-reg and Spatial-reg, respectively. Bottom panel: the comparison of DNN-reg and Spatial-reg by DCBC. Data are presented in mean +/- s.e.m.

We also used the DCBC to evaluate whether the border adjustment of the individual parcellation captured the specific features of individuals' functional connectivity patterns. We assumed that the deep-learn-based method (DNN-reg) should result in a higher DCBC than the direct spatial registration of MBMv4 (Spatial-reg). Figure 4D (Top panel) presents the functional connectivity for the pairs of vertices within the same parcel (average correlation values within the same surface length $0-4 \mathrm{~mm}$ were 0.8331 and 0.8172 for DNN-reg and Spatial-reg) and between different parcels (average correlation values were 0.8256 and 0.8171 for DNN-reg and Spatial-reg). Thus, the DNN-reg had higher DCBC (within-between) than the Spatial-reg (Fig. 4D, bottom panel; the average DCBC values were 0.0167 and 0.0085 for the DNN-reg and the Spatial-reg, respectively; multiple comparisons for One-Way ANOVA $\left.F_{(1,2512)}=20.35, p=6.74 \times 10^{-6}\right)$. In sum, the border adjustment by the proposed deep learning network reflects individual functional connectivity patterns. 


\section{MBMv4 reflects accurate functional and topographical organizations}

As evaluated from functional connectivity, MBMv4 provides a more accurate reflection of the functional parcellation of the cortex than current histology-based atlases. To further verify this reliability, we made a task activation map during the presentation of a fullscreen movie from our previous study (Tian et al., 2021), in which the visual field encompassed by the movie covered $10 \mathrm{deg} \times 8 \mathrm{deg}$. This activation map was then registered onto the same individual MBMv4 map and the histological-based Paxinos et al. (2012) atlas to examine the overlap between the activations and functional parcels. We found that the MBMv4 has a good correspondence with task activations by visual inspection, such as the co-activation of foveal V1, MT, and temporal parcels (Figure 5A). Additionally, measuring by the distances between the borders of task activation and the borders of functional parcels in the MBMv4 or the borders of brain regions in Paxinos atlas, we found that the parcel borders of MBMv4 have higher consistency with the activation map compared with Paxinos atlas (Fig. 5A, the scatterplots; Wilcoxon paired signed-rank test: Monkey ID 25, $N=878, p=3.07 \times 10^{-40}$ for the left hemisphere; $N=816$, $p=6.11 \times 10^{-26}$ for the right hemisphere. Monkey ID $15, N=826, p=2.22 \times 10^{-25}$ for the left hemisphere; $\mathrm{N}=850, \mathrm{p}=2.95 \times 10^{-53}$ for the right hemisphere). Therefore, MBMv4 reflects functional differences that cytoarchitectonics does not capture, possibly because the latter contains the full visual field representations. MBMv4 might be a helpful functional localizer that helps us enhance the cross-species studies (Russ et al., 2021). 

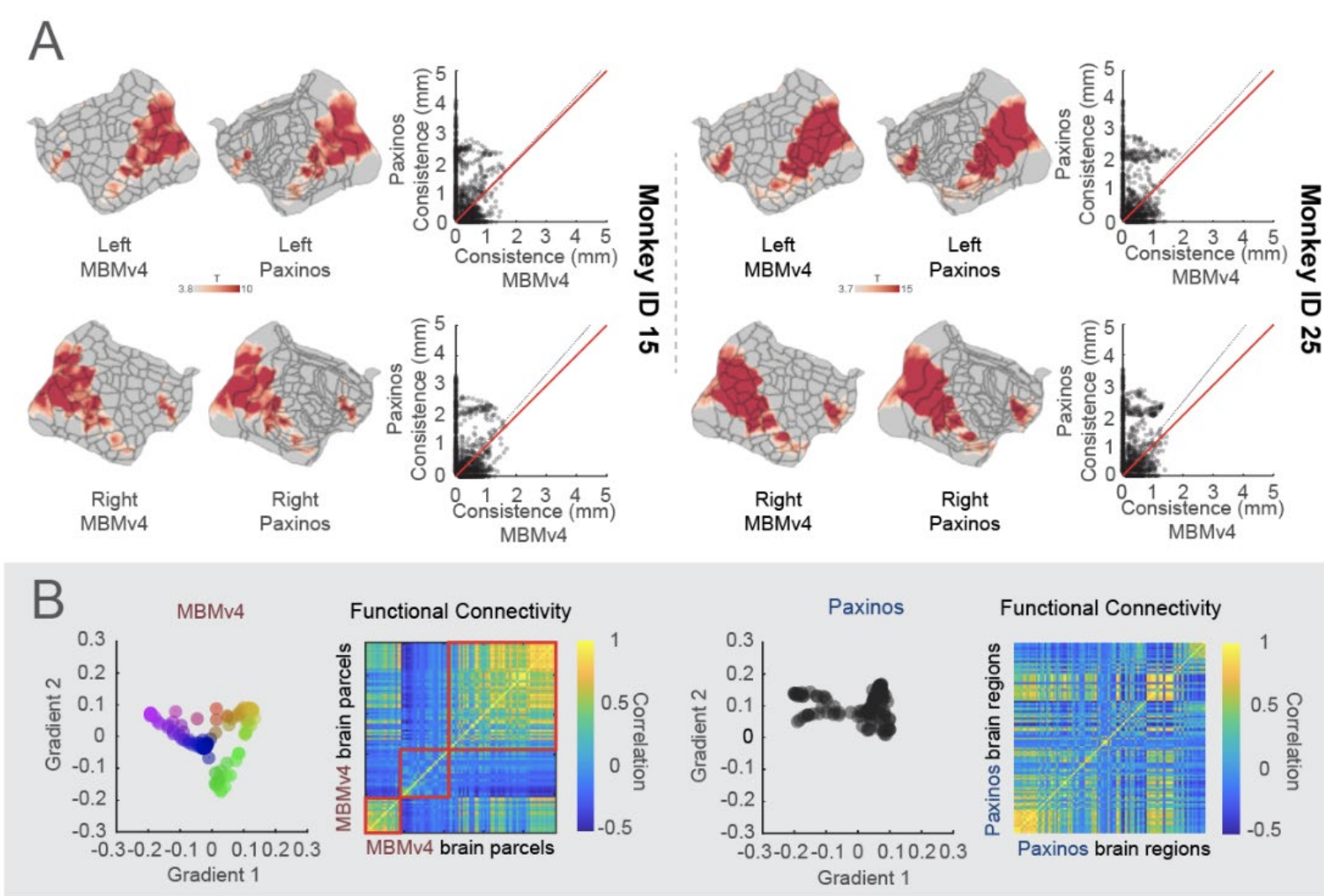

Figure 5. MBMv4 matches functional boundaries and preserves the topographical organization of the functional connectivity. (A) The visual task activation maps from two monkeys are overlaid on the parcel boundaries from individual MBMv4 parcellation and Paxinos atlas (Left panel: monkey ID 15; Right panel: monkey ID 25). The scatter plots compare the boundary matching of MBMv4 and Paxinos atlas with the activation maps, measured by the distance between the borders of the activation maps and the parcel/region borders of MBMv4 or Paxinos atlas. The dashed black line represents the diagonal line, and the red line represents the linear fitting line. (B) The scatter plots in the left panel are the first two axes of principal eigenvectors, decomposed by the functional connectivities of MBMv4 and Paxinos atlas (the spectrum colors denote the gradient position in this $2 D$ space). The heatmaps of functional connectivities sorted by the scores of the first axis (gradient 1) are shown in the right panel.

Besides the clear functional boundaries, MBMv4 also preserved the topographical organization of the functional connectivity. Recent methodological developments have 
allowed complex brain features to be mapped to the low-dimensional representations as gradients (Margulies et al., 2016), and these gradients characterized the topographical organization of the functional brain connectome from unimodal to trans-modal networks. If the atlas complies with the topographical organization, we should be able to identify such gradients. As shown in Fig. 5B left panel, MBMv4 results in a pattern of gradient spectrum for functional connectivity. In contrast, we did not find a gradient pattern based on the Paxinos et al. (2012) atlas (right panel in Fig. 5B). Therefore, MBMv4 offers an alternative view to understanding the functional connectome of the marmoset brain.

\section{MBMv4 is an essential link between the functional and structural connectivity}

Since MBMv4 offers a better scheme to study the functional connectome, it is worth linking it to structural information to investigate relationships between structural and functional connectivity. To investigate this, we used a whole-brain computational model (Deco et al., 2009; Deco et al., 2017; Ipina et al., 2020). The processing procedure is shown in Fig. 6A: we constructed the structural connectivity based on MBMv4 and Paxinos et al. (2012) atlas, using either the in-vivo diffusion MRI or ex-vivo ultra-highresolution diffusion MRI or neuronal tracing dataset. After simulating the neurodynamics of every functional parcel or brain region based on the structural connectivities, we obtained the whole-brain functional connectivity to compare with the empirical functional connectivity from the actual resting-state fMRI data. We used Pearson's correlation to measure the similarity between the simulated and the empirical functional connectivity. Moreover, we used group-average functional connectivity as an empirical observation for the ex-vivo diffusion MRI and neuronal tracing dataset, and individual functional connectivity for the individual in-vivo diffusion MRI. 


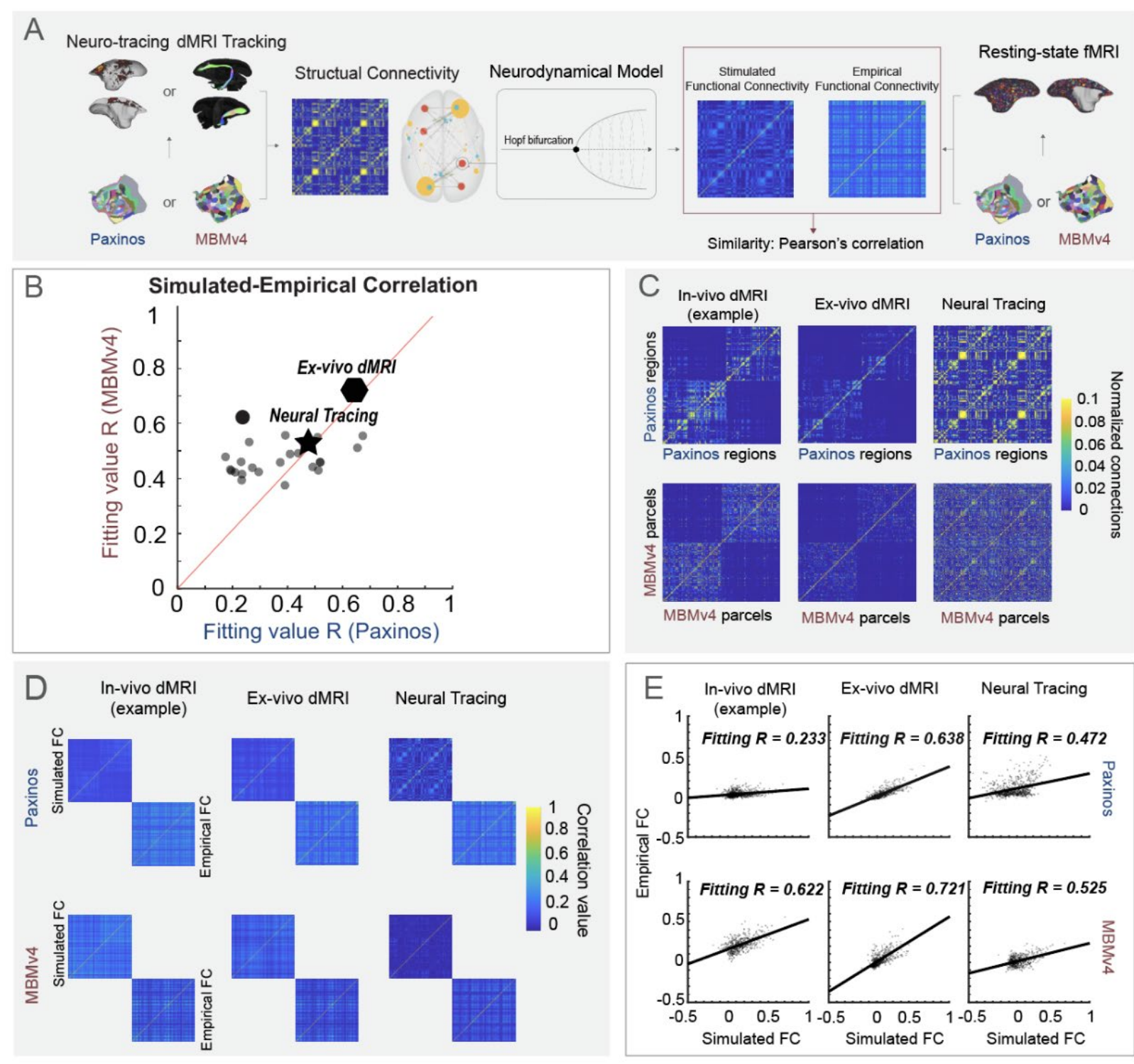

Figure 6. A computational framework links the structural-functional connectivity according to different parcellation. (A) The application of the whole-brain modeling, including the estimation of structural connectivity from the neuronal tracing or different types of diffusion MRI (in-vivo or ex-vivo) according to the Paxinos atlas or MBMv4, the simulation of functional connectivity from structural connectivity by the Hopf bifurcation neurodynamical functions, and the similarity measure with empirical connectivity from resting-state fMRI. (B) The comparison of the fitting effect based on Paxinos atlas and MBMv4 in different spatial scales. The round dot represents an example from individual in-vivo diffusion MRI, the polygon is from ex-vivo diffusion MRI, the star is from neuronal tracing, and the solid red line represents the diagonal line. (C) The estimated structural 
connectivity labeling in (B) according to the Paxinos atlas or MBMv4. (D) The simulated functional connectivity from structural connectivity $(C)$ and their empirical functional connectivity from actual data. (E). The correlation between the simulated and empirical functional connectivity from $(D)$, Solid black lines represent marginal regression lines.

The modeling results suggest that accurate estimation of structural connectivity is essential for the simulation of functional connectivity. The extra high-resolution ex-vivo diffusion MRI with the most detailed description of structural information (the polygon in Fig. 6B and results in Fig. 6C-E) resulted in the highest similarity for MBMv4 $(R=0.721)$ and the Paxinos atlas $(\mathrm{R}=0.638)$. On the other hand, due to the relatively low resolution of in-vivo diffusion MRI and individual differences, in-vivo diffusion MRI resulted in a considerable variety of simulations (all circles in Fig. 6B: the average fitting values from 25 animals were 0.4707 for MBMv4, and 0.3659 for Paxinos atlas, see an example with the best performance is in Fig. 6C-E). Whereas, based on the cellular connectivity from the aggregated neuronal tracing, we obtained a neutral performance with the correlation of 0.525 for MBMv4 and 0.472 for Paxinos atlas (the star in Fig. 6B and results in Fig. 6CE). However, no matter which data was used for structural connectivity estimation, we always found that the modeling predicted by MBMv4 fits the empirical functional data better than the Paxinos atlas (summary in Fig. 6B; Wilcoxon paired signed-rank test: $\mathrm{N}=27, \mathrm{p}=0.002947$ ). Therefore, MBMv4 preserves a reasonable organization from the examination of the structure-function relationship. Compared with cytoarchitectonics, MBMv4 provides essential complementary information about functional connectivity, which is helpful for us to investigate the discrepancy between structure and MRI-based function.

\section{Discussion}

There are a variety of challenges when trying to adapt well-established approaches for human neuroimaging to NHP neuroimaging (Milham et al., 2018). The present study used 
effective and practical training and imaging protocols to scan a large cohort of marmosets. Despite the different scanners in two institutes (7T and 9.4T), the protocol produced similar data quality, suggesting the compatibility of our approach (see method for details, the supplementary Fig. S1). Given that, we pool in-vivo resting-state fMRI dataset from two institutes (details in the supplementary table S1) to create the most comprehensive functional connectivity dataset of the marmoset brain, which was integrated with the invivo diffusion MRI of the same cohort, as well as the highest resolution ex-vivo diffusion MRI (Liu et al., 2020) and the most extensive mesoscale retrograde neuronal tracing (Majka et al., 2020) available to date. This resource allowed to expedite the mapping of marmoset brains and large-scale cross-species comparison.

\section{Improving functional connectivity mapping of the marmoset brain}

Like humans, the marmoset cerebral cortex is also composed of large-scale networks. However, the first awake resting-state fMRI study of the marmosets (Belcher et al., 2013) found only 12 functional networks (10 cortical networks). Later, another ICA study described 8 brain networks, possibly due to the influence of anesthesia (Ghahremani et al., 2017). Based on extensive awake rs-fMRI data to date, the present study mapped the large-scale functional networks and built the first network-based parcellation, providing a more comprehensive description of distinct functional networks in the marmoset brain, including a total of 19 brain networks (15 cortical networks and 4 subcortical networks in Fig. 1 and the supplementary Fig. S2). Moreover, based on functional-connectivity boundary maps, we also created a population-based cortical parcellation in a fine-scale (MBMv4; Fig. 4) with a total of 192 distinct parcels (96 per hemisphere). The previous study of the human cerebral cortex identified 422 discrete functional-connectivity parcels using the same approach, 206 in the left hemisphere and 216 in the right hemisphere (Gordon et al., 2016). However, since adopting the marmoset as a research model in neuroscience is relatively recent, the number of functional-connectivity parcels for the marmoset cortex has remained unknown. Therefore, our marmoset parcellation MBMv4 could be a helpful reference for the cross-species comparison. 
It is also worth noting that our functional parcels do not exactly correspond to the traditional cytoarchitectonic definition of the cortical areas (Amunts and Zilles, 2015; Kaas, 1987). Consistent with many brain parcellations by non-invasive neuroimaging (Glasser et al., 2016; Gordon et al., 2016; Kong et al., 2021; Schaefer et al., 2018; Van Essen and Glasser, 2018; Van Essen et al., 2012), our defined area-level functional parcels most likely reflect a different type of computational sub-units, in agreement with the idea that the brain is organized in multiple scales (Churchland and Sejnowski, 1988; van den Heuvel and Yeo, 2017). Therefore, compared with available structural atlases, MBMv4 captures the organization of functional connectivity accurately. For example, MBMv4 achieved better task correspondence (Fig. 5A), due to a strong link between task-fMRI and rs-fMRI (Mennes et al., 2010; Smith et al., 2009; Tavor et al., 2016). Another evidence is the topographical gradient organization of functional connectivity (Fig. 5B). Last is better modeling simulation linking with its structural connectivity (Fig. 6).

Consistent with the previous findings in humans (Glasser et al., 2016; Gordon et al., 2016; Van Essen and Glasser, 2018), MBMv4 still does not replicate cytoarchitectonic areas faithfully. For example, the somatomotor cortex is parcellated into subregions that appear to correspond to representations of the facial, forelimb, and trunk musculatures across multiple areas, and areas such as $\mathrm{V} 1$ and $\mathrm{V} 2$ are subdivided into many functional parcels according to eccentricity in visual field representation, which are contiguous across areas (Rosa and Tweedale, 2005), but may include discontinuities (Yu et al., 2020). Although previous studies also revealed that some topographically organized cytoarchitectonic areas could be dissociated with the resting-state functional responses (Long et al., 2014; Rao et al., 1995), yet, as we demonstrated before, the present MBMv4 should be considered a functional-connectivity description, providing complementary information about the organization that cannot be observed via anatomy.

\section{Improving functional mapping of the individual marmoset brain}

An essential goal of this study is to reflect individual characteristics by creating parcels from the individual subject data. Although the boundary map-derived parcels could be 
used for individual analysis, we found that the subject boundary maps had significant variations, and the reproducibility becomes lower than the group-level map (Fig. 3). This finding emphasizes the need to acquire large amounts of data for the reliable test-retest of the individual boundary map. Given that, we developed a deep neural network to map reliable population-level MBMv4 into every individual nonlinearly. As a result, we demonstrated good reliability in the test-retest dataset (across sessions from the same individual; see Fig. 4C) and the applications of task-fMRI activation mapping from the same individuals (Fig. 5A). Importantly, the locations of the most variable functional parcels are in the lateral prefrontal cortex and lateral temporal-occipital cortex (supplementary Fig. S5), corresponding to previously reported with exceptionally high inter-subject variability resting-state functional connectivities patterns (Mueller et al., 2013). Moreover, these regions co-locate with which expanded preferentially in primate evolution (Chaplin et al., 2013) and mature later in postnatal development (Sawiak et al., 2018). As the resting-state functional connectivities can be altered by many biological features, including development and associated with phenotypic correlations, a better understanding of the causes of intra-subject parcel variation will be our future work.

\section{Bridging the structural and functional connectome}

In addition to the functional connectome mapping, we integrated all currently available structural connectome datasets, including the in-vivo diffusion MRI, the ex-vivo highresolution diffusion MRI (Liu et al., 2020), and the extensive mesoscale tracing dataset (Majka et al., 2020). This allowed us to investigate the relationship between functional and structural connectivity with unprecedented detail. Using the whole-brain modeling (Deco et al., 2009; Deco et al., 2017), we observed the simulated functional connectivity from the structural connectivity based on MBMv4 had a higher coherence with empirical data, no matter which types of structural connectivity were used (Fig. 6B and examples Fig. 6C-E). The finding corroborates the conclusion that MBMv4 reflects meaningful computational sub-units from the view of whole-brain functional connectivity. Meanwhile, we also found room for modeling performance improvement by the detailed estimation of structural connectivity. For example, the ultra-high-resolution ex-vivo diffusion MRI data 
from a brain sample provides the most thorough structural information. Therefore, it has the best fitting results no matter which parcellation we use. Furthermore, since our modeling is simple with only two parameters that avoid overfitting simulation, the wholebrain model could be an efficient tool with broad applications to link structure and function for future studies.

\section{Limitations and Future directions}

Although we provided the most comprehensive multi-modal data resource for mapping the marmoset connectome, our current study still faced several limitations. First, the population used to generate the MBMv4 was sex-biased (31 males v.s. 8 females) due to the priority of colony expansion worldwide. Second, the neuronal tracing data were limited, not covering all cortical regions and missing subcortical information. Since the neuronal tracing data is directional, its intactness is helpful for the mapping of the structural connectome, and the accurate linkage between the structural and functional connectome in our next stage, which is the unidirectional diffusion tractography not capture. Finally, our parcellation only used the resting-state functional connectivity information as in many human studies (Glasser et al., 2016; Gordon et al., 2016; Kong et al., 2021; Schaefer et al., 2018). Thus, combining multiple task-fMRI data to improve functional parcellation becomes important in the future. Although we adopted the wellestablished approach from human studies, our multi-modal data and analyzing atlas tools will significantly accelerate the evolution of NHP neuroimaging research.

\section{Data and code availability}

\section{Data availability}

All NIH and ION resting-state fMRI, diffusion MRI, and neuronal tracing datasets are available at www.marmosetbrainmapping.org. The volume data are in NIFTI format, and the surface data are in CIFTI format. The raw MRI data are provided in both in-house data structure and the standard BIDS format for cross-platform sharing. In addition, data 
with different preprocessing, including a minimal preprocessing pipeline, are provided for analyzing purposes.

\section{Code availability}

The codes and analyzing pipelines (with code examples) used in this study are available at www.marmosetbrainmapping.org.

\section{Acknowledgments}

We thank Kaiwei Zhang and Binshi Bo at 9.4T core facility (CEBSIT) for assistance in data collection of the ION data, Lisa Zhang for the assistance in data collection of the NIH 7T data, Xiaojia Zhu for the assistance in organizing MRI data format, and the NIH Fellows Editorial Board for the editorial assistance. In addition, the following funding sources supported the study:

1) CL is supported by grants from the National Natural Science Foundation of China (No. 32171088) and the Shanghai Municipal Science and Technology Major Project (No. 2018SHZDZX05).

2) ZL is supported by grants from the National Natural Science Foundation of China (No. 81771821), CAS Pioneer Hundred Talents Program, Strategic Priority Research Program of the Chinese Academy of Sciences (No. XDB32030100), and the Shanghai Municipal Science and Technology Major Project (No. 2018SHZDZX05).

3) AS is supported by the Pennsylvania Department of Health Commonwealth Universal Research Enhancement (CURE.) Tobacco Settlement Appropriation - Phase 18 (Grant SAP4100083102) and NIH Intramural Research Programs CMS/LFMI/NINDS (ZIA NS003041).

4) MR is supported by the Australian Research Council (DP110101200, DP140101968, CE140100007) and International Neuroinformatics Coordinating Facility Seed Funding Grant. 
5) PM is supported by the National Science Centre (2019/35/D/NZ4/03031) and the International Neuroinformatics Coordinating Facility Seed Funding Grant.

6) GD is supported by a Spanish research project (ref. PID2019-105772GB-I00 AEI FEDER EU) funded by the Spanish Ministry of Science, Innovation, and Universities (MCIU), State Research Agency (AEI), and European Regional Development Funds (FEDER); HBP SGA3 Human Brain Project Specific Grant Agreement 3 (grant agreement no. 945539), funded by the EU H2020 FET Flagship program and SGR Research Support Group support (ref. 2017 SGR 1545), funded by the Catalan Agency for Management of University and Research Grants (AGAUR).

7) DG is supported by the NIMH and NINDS Intramural Research Programs (ZICMH002888) of the NIH (HHS, United States).

8) $\mathrm{CY}$ is supported by the Intramural Research Program of the NIH, National Institute of Neurological Disorders and Stroke.

\section{Author contributions}

$\mathrm{CL}, \mathrm{XT}, \mathrm{ZL}, \mathrm{AS}$, and MR designed and supervised the study; $\mathrm{YC}$ and $\mathrm{ZL}$ collected the ION MRI data; DS, CL, and XT collected the NIH MRI data; MR and PM collected the neuronal tracing data; CL, XT, CT, ZL, and KS preprocessed and organized the MRI data; $\mathrm{PM}, \mathrm{CL}, \mathrm{XT}$, and $\mathrm{HJ}$ preprocessed the neuronal tracing data; $\mathrm{CL}$ and $\mathrm{XT}$ constructed the functional-network maps; XT, CL, and MR constructed and evaluated the cortical parcellation maps; XT, CL, YP, and GD conducted the computational modeling; DG and $\mathrm{CL}$ implemented the atlas and resources into AFNI/SUMA. XT and CL wrote the original draft; and $C L, X T, A S, M R, P M, Z L, G D, C C$, and DG revised the draft. 


\section{Declaration of interests}

The authors declare no competing interests.

\section{Figure legends}

Figure 1. Identified cortical functional networks and their parcellation maps. The networks include (A) the ventral somatomotor, $(B)$ the dorsal somatomotor, $(C)$ the premotor, (D) the frontal pole, $(E)$ the orbital frontal cortex, $(F)$ the parahippocampus and temporal pole, (G-H) the auditory and salience-related network, (I-J) two trans-modal networks, which are most-likely related to the frontoparietal network and the defaultmode-network, and (K-O) the visual-related networks from the primary visual cortex to functional higher-level regions. These networks were combined to form two networkparcellation maps $(P-Q)$, which are dominated by the networks with short-range connectivity ( $P-Q$, top rows) and with long-range connectivity ( $P-Q$, bottom rows), respectively.

Figure 2. The functional connectivity boundary maps. (A). The population-based boundary maps from the $\mathrm{ION}$, the $\mathrm{NIH}$, and the combined datasets. These maps are highly consistent, with the average Dice's coefficient of 0.7. (B). Boundary maps in the left hemisphere from four exemplar monkeys (two from the NIH cohort and two from the ION, including the flagship marmosets). (C-D). The heatmap of the average Dice's coefficients for both hemispheres between individuals and its distribution histogram. (E). The change of the average Dice's coefficients for both hemispheres with the number of runs in the same individuals.

Figure 3. Marmoset Brain Mapping Atlas Version 4 (MBMv4). (A) The processing procedure includes generating the population functional-connectivity boundary maps, defining the local minima for seeding, and generating parcels by the "watershed" algorithm. (B) The resulting 96 functional-connectivity parcels per hemisphere overlaid on 
the white matter surface and flat map of MBMv3 (Liu et al., 2021). (C) The evaluation metric of distance-controlled boundary coefficient (DCBC). According to parcellations (Right panel: MBMv1, MBMv4, Paxinos, and RIKEN atlas), all pairs of voxels/vertices were categorized into "within" or "between" parcels (left panel), and the DCBC metric was calculated by the differences (within-between) in functional connectivity as the function of distance on the surface $(0-4 \mathrm{~mm}$ in steps of $0.5 \mathrm{~mm})$. Data are presented in mean $+/-$ s.e.m.

Figure 4. Mapping individual functional-connectivity parcellation. (A) An overview of individual mapping based on the deep neural network approach. (B) MBMv4 Mapping of each individual. Left panel: the concordance between the population MBMv4 and individual parcellations. Data are presented by the violin and the box plots (25th percentile and 75 percentile), in which the white point represents the average value; Right panel: three examples of individual parcellations. The underlay (color-coded) presents the population MBMv4, and the overlay (black border) shows the individual parcellations. (C) Mapping of MBMv4 per session. Left panel: The concordance between every individual parcellation and the corresponding parcellation using one session data; Right panel: representative parcellations of three sessions from one marmoset. The color-coded underlay represents individual parcellation, while the black border overlay shows the session-based parcellation. (D) The distance-controlled boundary coefficient (DCBC) for the individual parcellation generated by the spatial registration (Spatial-reg, blue) and the deep neural network (DNN-reg, red). Top panel: the functional connectivity for all pairs of vertices within the same parcel and between parcels for DNN-reg and Spatial-reg, respectively. Bottom panel: the comparison of DNN-reg and Spatial-reg by DCBC. Data are presented in mean +/- s.e.m.

Figure 5. MBMv4 matches functional boundaries and preserves the topographical organization of the functional connectivity. (A) The visual task activation maps from two monkeys are overlaid on the parcel boundaries from individual MBMv4 parcellation and Paxinos atlas (Left panel: monkey ID 15; Right panel: monkey ID 25). The scatter plots compare the boundary matching of MBMv4 and Paxinos atlas with the activation 
maps, measured by the distance between the borders of the activation maps and the parcel/region borders of MBMv4 or Paxinos atlas. The dashed black line represents the diagonal line, and the red line represents the linear fitting line. (B) The scatter plots in the left panel are the first two axes of principal eigenvectors, decomposed by the functional connectivities of MBMv4 and Paxinos atlas (the spectrum colors denote the gradient position in this 2D space). The heatmaps of functional connectivities sorted by the scores of the first axis (gradient 1 ) are shown in the right panel.

Figure 6. A computational framework links the structural-functional connectivity according to different parcellation. (A) The application of the whole-brain modeling, including the estimation of structural connectivity from the neuronal tracing or different types of diffusion MRI (in-vivo or ex-vivo) according to the Paxinos atlas or MBMv4, the simulation of functional connectivity from structural connectivity by the Hopf bifurcation neurodynamical functions, and the similarity measure with empirical connectivity from resting-state fMRI. (B) The comparison of the fitting effect based on Paxinos atlas and MBMv4 in different spatial scales. The round dot represents an example from individual in-vivo diffusion MRI, the polygon is from ex-vivo diffusion MRI, the star is from neuronal tracing, and the solid red line represents the diagonal line. (C) The estimated structural connectivity labeling in (B) according to the Paxinos atlas or MBMv4. (D) The simulated functional connectivity from structural connectivity (C) and their empirical functional connectivity from actual data. (E). The correlation between the simulated and empirical functional connectivity from (D), Solid black lines represent marginal regression lines.

\section{References}

Amunts, K., and Zilles, K. (2015). Architectonic Mapping of the Human Brain beyond Brodmann. Neuron 88, 1086-1107. 10.1016/j.neuron.2015.12.001.

Belcher, A.M., Yen, C.C., Stepp, H., Gu, H., Lu, H., Yang, Y., Silva, A.C., and Stein, E.A. (2013). Large-scale brain networks in the awake, truly resting marmoset monkey. J Neurosci 33, 1679616804. 10.1523/JNEUROSCI.3146-13.2013. 
Buckner, R.L., and Margulies, D.S. (2019). Macroscale cortical organization and a default-like apex transmodal network in the marmoset monkey. Nat Commun 10, 1976. 10.1038/s41467019-09812-8.

Buffalo, E.A., Movshon, J.A., and Wurtz, R.H. (2019). From basic brain research to treating human brain disorders INTRODUCTION. P Natl Acad Sci USA 116, 26167-26172.

10.1073/pnas.1919895116.

Churchland, P.S., and Sejnowski, T.J. (1988). Perspectives on cognitive neuroscience. Science 242, 741-745. 10.1126/science.3055294.

Cohen, A.L., Fair, D.A., Dosenbach, N.U., Miezin, F.M., Dierker, D., Van Essen, D.C., Schlaggar, B.L., and Petersen, S.E. (2008). Defining functional areas in individual human brains using resting functional connectivity MRI. Neuroimage 41, 45-57. 10.1016/j.neuroimage.2008.01.066. Deco, G., Jirsa, V., McIntosh, A.R., Sporns, O., and Kotter, R. (2009). Key role of coupling, delay, and noise in resting brain fluctuations. Proc Natl Acad Sci U S A 106, 10302-10307.

10.1073/pnas.0901831106.

Deco, G., Kringelbach, M.L., Jirsa, V.K., and Ritter, P. (2017). The dynamics of resting fluctuations in the brain: metastability and its dynamical cortical core. Sci Rep 7, 3095. 10.1038/s41598-017-03073-5.

Eliades, S.J., and Miller, C.T. (2017). Marmoset vocal communication: Behavior and neurobiology. Dev Neurobiol 77, 286-299. 10.1002/dneu.22464.

Ghahremani, M., Hutchison, R.M., Menon, R.S., and Everling, S. (2017). Frontoparietal Functional Connectivity in the Common Marmoset. Cereb Cortex 27, 3890-3905. 10.1093/cercor/bhw198.

Glasser, M.F., Coalson, T.S., Robinson, E.C., Hacker, C.D., Harwell, J., Yacoub, E., Ugurbil, K., Andersson, J., Beckmann, C.F., Jenkinson, M., et al. (2016). A multi-modal parcellation of human cerebral cortex. Nature 536, 171-178. 10.1038/nature18933.

Gordon, E.M., Laumann, T.O., Adeyemo, B., Huckins, J.F., Kelley, W.M., and Petersen, S.E. (2016). Generation and Evaluation of a Cortical Area Parcellation from Resting-State Correlations. Cereb Cortex 26, 288-303. 10.1093/cercor/bhu239.

Hacker, C.D., Laumann, T.O., Szrama, N.P., Baldassarre, A., Snyder, A.Z., Leuthardt, E.C., and Corbetta, M. (2013). Resting state network estimation in individual subjects. Neuroimage 82, 616-633. 10.1016/j.neuroimage.2013.05.108.

Homman-Ludiye, J., and Bourne, J.A. (2017). The marmoset: An emerging model to unravel the evolution and development of the primate neocortex. Dev Neurobiol 77, 263-272.

10.1002/dneu.22425.

Hudetz, A.G. (2012). General anesthesia and human brain connectivity. Brain Connect 2, 291302. 10.1089/brain.2012.0107.

Ipina, I.P., Kehoe, P.D., Kringelbach, M., Laufs, H., Ibanez, A., Deco, G., Perl, Y.S., and Tagliazucchi, E. (2020). Modeling regional changes in dynamic stability during sleep and wakefulness. Neuroimage 215, 116833. 10.1016/j.neuroimage.2020.116833.

Kaas, J.H. (1987). The organization of neocortex in mammals: implications for theories of brain function. Annu Rev Psychol 38, 129-151. 10.1146/annurev.ps.38.020187.001021.

King, M., Hernandez-Castillo, C.R., Poldrack, R.A., Ivry, R.B., and Diedrichsen, J. (2019). Functional boundaries in the human cerebellum revealed by a multi-domain task battery. Nat Neurosci 22, 1371-1378. 10.1038/s41593-019-0436-x. 
Kong, R., Yang, Q., Gordon, E., Xue, A., Yan, X., Orban, C., Zuo, X.N., Spreng, N., Ge, T., Holmes, A., et al. (2021). Individual-Specific Areal-Level Parcellations Improve Functional Connectivity Prediction of Behavior. Cereb Cortex 31, 4477-4500. 10.1093/cercor/bhab101. Liu, C., Ye, F.Q., Newman, J.D., Szczupak, D., Tian, X., Yen, C.C., Majka, P., Glen, D., Rosa, M.G.P., Leopold, D.A., and Silva, A.C. (2020). A resource for the detailed 3D mapping of white matter pathways in the marmoset brain. Nat Neurosci 23, 271-280. 10.1038/s41593-019-0575-0. Liu, C., Ye, F.Q., Yen, C.C., Newman, J.D., Glen, D., Leopold, D.A., and Silva, A.C. (2018). A digital 3D atlas of the marmoset brain based on multi-modal MRI. Neuroimage 169, 106-116. 10.1016/j.neuroimage.2017.12.004. Liu, C., Yen, C.C., Szczupak, D., Tian, X., Glen, D., and Silva, A.C. (2021). Marmoset Brain Mapping V3: Population multi-modal standard volumetric and surface-based templates. Neuroimage 226, 117620. 10.1016/j.neuroimage.2020.117620. Liu, C., Yen, C.C., Szczupak, D., Ye, F.Q., Leopold, D.A., and Silva, A.C. (2019). Anatomical and functional investigation of the marmoset default mode network. Nat Commun 10, 1975. 10.1038/s41467-019-09813-7.

Long, X., Goltz, D., Margulies, D.S., Nierhaus, T., and Villringer, A. (2014). Functional connectivity-based parcellation of the human sensorimotor cortex. Eur J Neurosci 39, 13321342. 10.1111/ejn.12473.

Majka, P., Bai, S., Bakola, S., Bednarek, S., Chan, J.M., Jermakow, N., Passarelli, L., Reser, D.H., Theodoni, P., Worthy, K.H., et al. (2020). Open access resource for cellular-resolution analyses of corticocortical connectivity in the marmoset monkey. Nat Commun 11, 1133.

10.1038/s41467-020-14858-0.

Majka, P., Bednarek, S., Chan, J.M., Jermakow, N., Liu, C., Saworska, G., Worthy, K.H., Silva, A.C., Wojcik, D.K., and Rosa, M.G.P. (2021). Histology-Based Average Template of the Marmoset Cortex With Probabilistic Localization of Cytoarchitectural Areas. Neuroimage 226, 117625. 10.1016/j.neuroimage.2020.117625.

Majka, P., Chaplin, T.A., Yu, H.H., Tolpygo, A., Mitra, P.P., Wojcik, D.K., and Rosa, M.G. (2016). Towards a comprehensive atlas of cortical connections in a primate brain: Mapping tracer injection studies of the common marmoset into a reference digital template. J Comp Neurol 524, 2161-2181. 10.1002/cne.24023.

Margulies, D.S., Ghosh, S.S., Goulas, A., Falkiewicz, M., Huntenburg, J.M., Langs, G., Bezgin, G., Eickhoff, S.B., Castellanos, F.X., Petrides, M., et al. (2016). Situating the default-mode network along a principal gradient of macroscale cortical organization. Proc Natl Acad Sci U S A 113, 12574-12579. 10.1073/pnas.1608282113.

Matsuzaki, M., and Ebina, T. (2020). Common marmoset as a model primate for study of the motor control system. Curr Opin Neurobiol 64, 103-110. 10.1016/j.conb.2020.02.013.

Mennes, M., Kelly, C., Zuo, X.N., Di Martino, A., Biswal, B.B., Castellanos, F.X., and Milham, M.P. (2010). Inter-individual differences in resting-state functional connectivity predict task-induced BOLD activity. Neuroimage 50, 1690-1701. 10.1016/j.neuroimage.2010.01.002.

Messinger, A., Sirmpilatze, N., Heuer, K., Loh, K.K., Mars, R.B., Sein, J., Xu, T., Glen, D., Jung, B., Seidlitz, J., et al. (2021). A collaborative resource platform for non-human primate neuroimaging. Neuroimage 226, 117519. 10.1016/j.neuroimage.2020.117519. 
Milham, M., Petkov, C., Belin, P., Ben Hamed, S., Evard, H., Fair, D.A., Fox, A., Basso, M.A., Belin, P., Kastner, S., et al. (2021). Toward next-generation primate neuroscience: A collaborationbased strategic plan for integrative neuroimaging. Neuron. 10.1016/j.neuron.2021.10.015. Milham, M., Petkov, C.I., Margulies, D., Schroeder, C., Basso, M., Belin, P., Fair, D.A., Fox, A., Kastner, S., Mars, R.B., et al. (2020). Accelerating the Evolution of Nonhuman Primate Neuroimaging. Neuron 105, 600-603. 10.1016/j.neuron.2019.12.023.

Milham, M.P., Ai, L., Koo, B., Xu, T., Amiez, C., Balezeau, F., Baxter, M.G., Blezer, E.L.A., Brochier, T., Chen, A., et al. (2018). An Open Resource for Non-human Primate Imaging. Neuron 100, 6174 e62. 10.1016/j.neuron.2018.08.039.

Mitchell, J.F., and Leopold, D.A. (2015). The marmoset monkey as a model for visual neuroscience. Neurosci Res 93, 20-46. 10.1016/j.neures.2015.01.008.

Mueller, S., Wang, D., Fox, M.D., Yeo, B.T., Sepulcre, J., Sabuncu, M.R., Shafee, R., Lu, J., and Liu, $\mathrm{H}$. (2013). Individual variability in functional connectivity architecture of the human brain. Neuron 77, 586-595. 10.1016/j.neuron.2012.12.028.

Okano, H. (2021). Current Status of and Perspectives on the Application of Marmosets in Neurobiology. Annu Rev Neurosci 44, 27-48. 10.1146/annurev-neuro-030520-101844.

Okano, H., and Mitra, P. (2015). Brain-mapping projects using the common marmoset. Neurosci Res 93, 3-7. 10.1016/j.neures.2014.08.014.

Rao, S.M., Binder, J.R., Hammeke, T.A., Bandettini, P.A., Bobholz, J.A., Frost, J.A., Myklebust, B.M., Jacobson, R.D., and Hyde, J.S. (1995). Somatotopic mapping of the human primary motor cortex with functional magnetic resonance imaging. Neurology 45, 919-924.

10.1212/wnl.45.5.919.

Rosa, M.G., and Tweedale, R. (2005). Brain maps, great and small: lessons from comparative studies of primate visual cortical organization. Philos Trans R Soc Lond B Biol Sci 360, 665-691. 10.1098/rstb.2005.1626.

Russ, B.E., Petkov, C.I., Kwok, S.C., Zhu, Q., Belin, P., Vanduffel, W., and Hamed, S.B. (2021). Common functional localizers to enhance NHP \& cross-species neuroscience imaging research. Neuroimage 237, 118203. 10.1016/j.neuroimage.2021.118203.

Sawiak, S.J., Shiba, Y., Oikonomidis, L., Windle, C.P., Santangelo, A.M., Grydeland, H., Cockcroft, G., Bullmore, E.T., and Roberts, A.C. (2018). Trajectories and Milestones of Cortical and Subcortical Development of the Marmoset Brain From Infancy to Adulthood. Cereb Cortex 28, 4440-4453. 10.1093/cercor/bhy256.

Schaefer, A., Kong, R., Gordon, E.M., Laumann, T.O., Zuo, X.N., Holmes, A.J., Eickhoff, S.B., and Yeo, B.T.T. (2018). Local-Global Parcellation of the Human Cerebral Cortex from Intrinsic Functional Connectivity MRI. Cereb Cortex 28, 3095-3114. 10.1093/cercor/bhx179. Schultz-Darken, N., Braun, K.M., and Emborg, M.E. (2016). Neurobehavioral development of common marmoset monkeys. Dev Psychobiol 58, 141-158. 10.1002/dev.21360.

Smith, S.M., Beckmann, C.F., Andersson, J., Auerbach, E.J., Bijsterbosch, J., Douaud, G., Duff, E., Feinberg, D.A., Griffanti, L., Harms, M.P., et al. (2013). Resting-state fMRI in the Human Connectome Project. Neuroimage 80, 144-168. 10.1016/j.neuroimage.2013.05.039. Smith, S.M., Fox, P.T., Miller, K.L., Glahn, D.C., Fox, P.M., Mackay, C.E., Filippini, N., Watkins, K.E., Toro, R., Laird, A.R., and Beckmann, C.F. (2009). Correspondence of the brain's functional architecture during activation and rest. Proc Natl Acad Sci U S A 106, 13040-13045. 10.1073/pnas.0905267106. 
Tavor, I., Parker Jones, O., Mars, R.B., Smith, S.M., Behrens, T.E., and Jbabdi, S. (2016). Task-free MRI predicts individual differences in brain activity during task performance. Science 352, 216220. 10.1126/science.aad8127.

Thiebaut de Schotten, M., Croxson, P.L., and Mars, R.B. (2019). Large-scale comparative neuroimaging: Where are we and what do we need? Cortex 118, 188-202.

10.1016/j.cortex.2018.11.028.

Tian, X., Silva, A.C., and Liu, C. (2021). The Brain Circuits and Dynamics of Curiosity-Driven Behavior in Naturally Curious Marmosets. Cereb Cortex 31, 4220-4232.

10.1093/cercor/bhab080.

van den Heuvel, M.P., and Yeo, B.T.T. (2017). A Spotlight on Bridging Microscale and Macroscale Human Brain Architecture. Neuron 93, 1248-1251. 10.1016/j.neuron.2017.02.048. Van Essen, D.C., and Glasser, M.F. (2018). Parcellating Cerebral Cortex: How Invasive Animal Studies Inform Noninvasive Mapmaking in Humans. Neuron 99, 640-663.

10.1016/j.neuron.2018.07.002.

Van Essen, D.C., Glasser, M.F., Dierker, D.L., Harwell, J., and Coalson, T. (2012). Parcellations and hemispheric asymmetries of human cerebral cortex analyzed on surface-based atlases. Cereb Cortex 22, 2241-2262. 10.1093/cercor/bhr291.

Walker, J., MacLean, J., and Hatsopoulos, N.G. (2017). The marmoset as a model system for studying voluntary motor control. Dev Neurobiol 77, 273-285. 10.1002/dneu.22461.

Wig, G.S., Laumann, T.O., and Petersen, S.E. (2014). An approach for parcellating human cortical areas using resting-state correlations. Neuroimage 93 Pt 2, 276-291.

10.1016/j.neuroimage.2013.07.035.

Woodward, A., Hashikawa, T., Maeda, M., Kaneko, T., Hikishima, K., Iriki, A., Okano, H., and Yamaguchi, Y. (2018). The Brain/MINDS 3D digital marmoset brain atlas. Sci Data 5, 180009. 10.1038/sdata.2018.9.

Yu, H.H., Rowley, D.P., Price, N.S.C., Rosa, M.G.P., and Zavitz, E. (2020). A twisted visual field map in the primate dorsomedial cortex predicted by topographic continuity. Sci Adv 6.

10.1126/sciadv.aaz8673. 


\section{Methods}

\section{Data Collection and Preprocessing}

\section{Animals and MRI scanning}

The experimental procedures were approved by the Animal Care and Use Committees from the Institute of Neuroscience (ION) at the Chinese Academy of Sciences and National Institute of Neurological Disorders and Stroke at the National Institutes of Health $(\mathrm{NIH})$. The data acquisition procedure from both centers followed the same animal training protocol, 8-element radiofrequency (RF) coil design (Papoti et al., 2017), and MRI scanning protocols.

Thirteen marmosets (12 males and 1 female) were recruited from the ION cohort, from which we generated 62 awake resting fMRI sessions and 351 runs (17 min per run). Twenty-six marmosets (19 males and 7 females) were recruited from the $\mathrm{NIH}$ cohort to produce 48 awake resting-state fMRI sessions and 358 runs. Therefore, the NIH data and ION data had a comparable number of runs. The two datasets included 39 marmosets with 110 sessions, $709 \mathrm{fMRI}$ runs, and 12053 mins total scan time. The detailed demographic information is provided in Supplementary Table 1. All marmosets underwent a 3-to-4 week acclimatization protocol as previously described (Silva et al., 2011). After completing the training, all marmosets were properly acclimated to laying in the sphinx position in an MRI-compatible cradle. Their heads were comfortably restrained with 3D-printed anatomically conforming helmets that allowed the resting-state $\mathrm{fMRI}(\mathrm{rs}-\mathrm{fMRI})$ data acquisition as the animals lay relaxed in their natural resting position. 
bioRxiv preprint doi: https://doi.org/10.1101/2021.11.12.468389; this version posted November 13, 2021. The copyright holder for this preprint (which was not certified by peer review) is the author/funder, who has granted bioRxiv a license to display the preprint in perpetuity. It is made All 39 marmosets were imaged using identical rs-fMRI protocols and pulse sequences, except for a minor adjustment in the echo time (TE) made to accommodate hardware differences between the ION and the NIH gradient sets. The ION marmosets were scanned in a $9.4 \mathrm{~T} / 30 \mathrm{~cm}$ horizontal MRI scanner (Bruker, Billerica, USA) equipped with a $20 \mathrm{~cm}$ gradient set capable of 300 $\mathrm{mT} / \mathrm{m}$ gradient strength. The scanner was fitted with a $154 \mathrm{~mm}$ ID quadrature RF coil used for signal excitation and an 8-channel phased-array RF coil (Papoti et al., 2017) custom-built for marmosets (Fine Instrument Technology, Brazil). Multiple runs of rs-fMRI data were collected in ParaVision 6.0.1 software using a 2D gradient-echo (GE) EPI sequence with the following parameters: TR $=2$ $\mathrm{s}, \mathrm{TE}=18 \mathrm{~ms}$, flip angle $=70.4^{\circ}, \mathrm{FOV}=28 \times 36 \mathrm{~mm}$, matrix size $=56 \times 72,38$ axial slices, slice thickness $=0.5 \mathrm{~mm}, 512$ volumes $(17 \mathrm{~min})$ per run. The GEEPI fMRI data were collected using two opposite phase-encoding directions (LR and RL) to compensate for EPI distortions and signal dropouts. Two sets of spin-echo EPI with opposite phase-encoding directions (LR and RL) were also collected for EPI-distortion correction $(T R=3000 \mathrm{~ms}, \mathrm{TE}=37.69 \mathrm{~ms}$, flip angle $=90^{\circ}, \mathrm{FOV}=28 \times 36 \mathrm{~mm}$, matrix size $=56 \times 72,38$ axial slices, slice thickness $=0.5 \mathrm{~mm}, 8$ volumes for each set). After each rs-fMRI session, a T2weighted structural image $\left(\mathrm{TR}=8000 \mathrm{~ms}, \mathrm{TE}=10 \mathrm{~ms}\right.$, flip angle $=90^{\circ}, \mathrm{FOV}=$ $28 \times 36 \mathrm{~mm}$, matrix size $=112 \times 144,38$ axial slices, slice thickness $=0.5 \mathrm{~mm}$ ) was scanned for co-registration purposes.

The NIH marmosets were scanned in a $7 \mathrm{~T} / 30 \mathrm{~cm}$ horizontal MRI (Bruker, Billerica, USA) equipped with a $15 \mathrm{~cm}$ customized gradient set capable of 450 $\mathrm{mT} / \mathrm{m}$ gradient strength (Resonance Research Inc., Billerica, USA). The scanner was fitted with a $110 \mathrm{~mm}$ ID linear RF coil used for signal excitation and an 8-channel phased-array RF coil custom-built for marmosets (Papoti et al., 2017). During each scanning session, multiple runs of rs-fMRI data were collected in ParaVision 6.0.1. software using a 2D gradient-echo (GE) EPI sequence with the following parameters: $T R=2 \mathrm{~s}, T E=22.2 \mathrm{~ms}$, flip 
bioRxiv preprint doi: https://doi.org/10.1101/2021.11.12.468389; this version posted November 13, 2021. The copyright holder for this preprint (which was not certified by peer review) is the author/funder, who has granted bioRxiv a license to display the preprint in perpetuity. It is made angle $=70.4^{\circ}, \mathrm{FOV}=28 \times 36 \mathrm{~mm}$, matrix size $=56 \times 72,38$ axial slices, slice thickness $=0.5 \mathrm{~mm}, 512$ volumes $(17 \mathrm{~min})$ per run. The GE-EPI fMRI data were collected using two opposite phase-encoding directions ( $L R$ and $R L$ ) to compensate for EPI distortions and signal dropouts. Two sets of spin-echo EPI with opposite phase-encoding directions (LR and $R L$ ) were also collected for EPI-distortion correction $\left(\mathrm{TR}=3000 \mathrm{~ms}, \mathrm{TE}=36 \mathrm{~ms}\right.$, flip angle $=90^{\circ}, \mathrm{FOV}=28$ $\times 36 \mathrm{~mm}$, matrix size $=56 \times 72,38$ axial slices, slice thickness $=0.5 \mathrm{~mm}, 8$ volumes for each set). After each rs-fMRI session, a T2-weighted structural image $\left(T R=6000 \mathrm{~ms}, \mathrm{TE}=9 \mathrm{~ms}\right.$, flip angle $=90^{\circ}, \mathrm{FOV}=28 \times 36 \mathrm{~mm}$, matrix size $=112 \times 144,38$ axis slices, slice thickness $=0.5 \mathrm{~mm}$ ) was scanned for coregistration purposes. Furthermore, multishell diffusion MRI (DTI) datasets were collected using a 2D diffusion-weighted spin-echo EPI sequence with the following parameters: TR $=5.1 \mathrm{~s}, \mathrm{TE}=38 \mathrm{~ms}$, number of segments $=88, \mathrm{FOV}$ $=36 \times 28 \mathrm{~mm}$, matrix size $=72 \times 56$, slice thickness $=0.5 \mathrm{~mm}$, a total of 400 DWI images for two-phase encodings (blip-up and blip-down) and each has 3 $b$ values $(8 b=0,64 b=2400$, and $128 b=4800)$, and the scanning duration was about $34 \mathrm{~min}$. The multishell gradient sampling scheme was generated using the Q-shell sampling method (Caruyer et al., 2013).

\section{Data Preprocessing}

The rs-fMRI datasets were preprocessed by the customized script involving AFNI (Cox, 2012), FSL (Jenkinson et al., 2012), ANTs (Tustison et al., 2011), and Connectome Workbench (Marcus et al., 2011). In brief, the rs-fMRI data were slice-timing-corrected and motion-corrected by the "3dTshift" and "3dvolreg" commands of AFNI, and corrected for EPI distortions by the "top-up" command of FSL. The rs-fMRI datasets were further preprocessed by regressing linear and quadratic trends, demeaning, and censoring for motion using derivatives of motion parameters and motion-sensor regressors (any TRs and the previous TRs were censored if the detection motion was $>0.2 \mathrm{~mm}$ ). White matter and cerebrospinal fluid signal were removed, and the rs-fMRI 
bioRxiv preprint doi: https://doi.org/10.1101/2021.11.12.468389; this version posted November 13, 2021. The copyright holder for this preprint (which was not certified by peer review) is the author/funder, who has granted bioRxiv a license to display the preprint in perpetuity. It is made datasets were band-pass filtered $(0.01-0.1 \mathrm{~Hz})$. The above nuisance signal regression and band-passing filtering were carried out by the "3dDeconvolve" and "3dTproject" commands in AFNI. Next, the preprocessed data were spatially normalized to the template space of our Marmoset Brain Atlas Version3 (MBMv3) by the "antsRegistration" routine of ANTs (Liu et al., 2021). The spatial normalization concatenated multiple transformations, including 1) rigidbody transformation of each fMRI run to the T2-weighted image acquired at the end of each session, 2) rigid-body transformation of T2-weighted images from each session to a cross-session averaged T2-weighted image from each animal, 3) affine and nonlinear transformation of the averaged T2-weighted image from each animal to the T2w template of our MBMv3 space. Finally, all preprocessed data were mapped to 3D brain surfaces using the Connectome Workbench.

The in-vivo diffusion MRI dataset was preprocessed by the DIFF_PREP, DR_BUDDI, and DR_TAMAS pipelines of TORTOISE (Pierpaoli, 2010). The DIFF_PREP and DR_BUDDI routines incorporated correction for eddycurrents- and EPI-induced distortions using pairs of diffusion data acquired with opposite phase encoding (blip-up and blip-down) and the T2-weighted image and merging the preprocessed pairs into one dataset. The nonlinear spatial registration from the individual space to the DTI template of our MBMv3 space (Liu et al., 2021) was carried out using the DR_TAMAS routine of TORTOISE. The registration information was then used to transform multiple atlases to the individual space for diffusion tractography.

The neuronal tracing data were mapped onto the histological NM template from our previous study (Majka et al., 2021). The NM template is a population-based 3D cortical template generated from Nissl-stained serial sections of 20 marmosets. Since the NM template only covers the cortex and has Nissl-stain contrast and a $75 \mu \mathrm{m}$ isotropic high spatial resolution, its direct spatial 
bioRxiv preprint doi: https://doi.org/10.1101/2021.11.12.468389; this version posted November 13, 2021. The copyright holder for this preprint (which was not certified by peer review) is the author/funder, who has granted bioRxiv a license to display the preprint in perpetuity. It is made transformation to our in-vivo MBMv3 template is inaccurate. Thus, we modified the $80 \mu \mathrm{m}$ isotropic ultra-high-resolution MTR template of our Marmoset Brain Atlas Version-2 (MBMv2) atlas (Liu et al., 2020) to remove the parts of the brain that were not covered in the NM template, including the cerebellum, brainstem, and parts of subcortical structures. This step increased the accuracy of registration between the NM template and the MBMv2 template. Then, the exvivo MTR template of the MBMv2 was nonlinearly transformed to the in-vivo myelin-map template of our MBMv3. By concatenating the two transformations (the NM-to-MBMv2 and the MBMv2-to-MBMv3), we accurately converted the neuronal tracing data from the NM template to the MRI template. We then mapped the neuronal tracing data onto the MBMv3 cortical surfaces.

\section{Functional Networks, Cortical Parcellation and Network Modeling}

\section{Brain network identification by the Group-ICA}

Independent Component Analysis (ICA) was performed by the Group-ICA routine of the GIFTI software (https://trendscenter.org/software/gift/) to identify the brain networks using a number of different component settings. First, preprocessed data without regression of nuisance covariates were group-ICA analyzed with increasing numbers of ICA components from 20 to 80 in steps of 10. We tested the reliability of different ICA methods, including the default "Infomax" ICA algorithm or "ICASSO" group-ICA method, on different datasets (the NIH dataset, the ION dataset, or combined both datasets) and obtained consistent results regardless of the ICA setting or dataset used. Every resulting component from Group-ICA analyses was visually inspected and sorted according to its neuroanatomical features. Since the sorted elements were highly consistent across different settings of ICA-component numbers, we selected the best component to represent every labeled network. We identify 
bioRxiv preprint doi: https://doi.org/10.1101/2021.11.12.468389; this version posted November 13, 2021. The copyright holder for this preprint (which was not certified by peer review) is the author/funder, who has granted bioRxiv a license to display the preprint in perpetuity. It is made 19 functional resting-state networks comprising of 15 cortical networks and 4 subcortical networks (Fig. 1 A-O and Supplementary Fig. S2).

We combined the 15 cortical networks according to their normalized $Z$ scores from ICA to create a cortical-network parcellation. Due to local connectivity being stronger than long-range connectivity, the primary map (Fig. 1P-Q, top rows) is dominated by the short-range networks (i.e., Fig. $1 \mathrm{G}, \mathrm{I}, \mathrm{J}, \mathrm{K}, \mathrm{L}, \mathrm{I}$, and O). Thus, we created the second one (Fig. 1P-Q, bottom rows) to cover the long-range connectivity that was not captured by the primary map.

\section{Boundary map generation}

Following similar procedures to the ones described previously in a human imaging study (Gordon et al., 2016), the boundary mapping of resting-state functional connectivity data was implemented in the Connectome Workbench and using customized Matlab codes (Mathworks, Natick, USA, Version 2019b; see the scripts in our open resource). First, the time course of every surface vertex for each brain hemisphere of each subject was correlated with every other surface vertex to make a correlation map. Then, a similarity map was created for every vertex by calculating pairwise spatial correlations between all correlation maps. Thirdly, the first spatial derivative was applied on the similarity map by the Connectome Workbench's function "cifti-gradient" to generate gradient maps for each brain hemisphere of each subject. Next, the gradient maps were averaged across subjects to produce the group gradient maps for each brain hemisphere. Lastly, the "watershed by flooding" algorithm was applied to identify boundaries in the gradient maps.

\section{Test-retest evaluation of the boundary map}

To compare the reliability of the boundary maps between the ION and the $\mathrm{NIH}$ datasets (Fig. 2A and supplementary Fig. S3), between the individuals (Fig. 2C-E) and between runs from the same individual (Fig. 2E), we first thresholded 
bioRxiv preprint doi: https://doi.org/10.1101/2021.11.12.468389; this version posted November 13, 2021. The copyright holder for this preprint (which was not certified by peer review) is the author/funder, who has granted bioRxiv a license to display the preprint in perpetuity. It is made two resulting boundary maps for each hemisphere to retain the cortical vertices most likely to be boundaries (i.e., retaining the top quartile of boundary values for a cumulative probability of 0.75 ) and assessed the overlap of the two thresholded boundaries by calculating the Dice's coefficient. The Dice similarity coefficient of two thresholded boundaries, A and B, is expressed as:

$$
\operatorname{dice}(A, B)=2 *\left|\frac{\text { intersection }(A, B)}{|A|+|B|}\right|
$$

The average Dice similarity coefficient is the mean of Dice similarity coefficients across hemispheres.

\section{Cortical parcellation based on the population-level boundary map}

The creation of parcels was implemented by the customized Matlab scripts (see our open resource). Firstly, based on the vertices with values smaller than their neighbors that were $<5$ vertices away, we identified all local minima of vertices on the boundary map as seeds for parcel creation. Then, the parcels were grown from these seeds using the "watershed algorithm" procedure as above, allowing them to expand outward from the seed until they met other parcels. Because the whole process depends on the number of seeds for parcel creation, this might result in a large number of parcels. Therefore, according to the performance, we manually defined a threshold for merging adjacent parcels, which is the 60th percentile of the values in the boundary map. It means that any two adjacent parcels with an average value below this threshold were considered not sufficiently dissimilar and should be merged. Finally, according to the population-level boundary map, we visually examined remaining parcels to identify those that needed further adjustment, including eliminating vertices and spatial smoothing. The process of parcel creation was semi-automated (Fig. 3A). We defined the resulting cortical parcellation with 96 functional 
bioRxiv preprint doi: https://doi.org/10.1101/2021.11.12.468389; this version posted November 13, 2021. The copyright holder for this preprint (which was not certified by peer review) is the author/funder, who has granted bioRxiv a license to display the preprint in perpetuity. It is made parcels in each hemisphere as our Marmoset Brain Mapping Atlas Version-4 (MBMv4) in Fig. 3B.

\section{Evaluation of cortical parcellation by the distance-controlled boundary coefficient (DCBC)}

Following a previous study (King et al., 2019), we used the distance-controlled boundary coefficient (DCBC) as a metric to evaluate functional boundaries between our parcels. The rationale for this method is that any two points belonging to any given parcel should have more similar functional profiles than those belonging to different parcels. Furthermore, because the functional organization varies smoothly, the correlation between two points will weaken with increasing spatial distance. Thus, we calculated the correlation coefficients for all pairs of points separated by a specific surface Euclidean distance, using $0.5 \mathrm{~mm}$ spatial bins (same as $\mathrm{fMRI}$ imaging spatial resolution) ranging from 0 to $4 \mathrm{~mm}$ for pairs of points residing within parcels or across different parcels (between). The DCBC defines the difference between the within-parcel and between-parcel pair correlations. A higher DCBC reflects that pairs within the same region are more functional, serving as a global parcellation measure. For the group comparison across atlases (Fig. $3 \mathrm{C}$ ), the DCBC metrics were calculated for each participant in each spatial bin and then averaged. For the same participant comparison across atlases (Fig. 4D), the DCBC metrics were calculated for each session in each spatial bin and then averaged.

\section{Comparison with alternative atlases}

We compared our parcellation against alternative digital parcellations created by various approaches. These alternative parcellations included: (1) Paxinos atlas (Paxinos et al., 2012), the most commonly used atlas in marmoset brain research, which is cytoarchitectonic characterization by immunohistochemical sections, and here we used its 3D digital version (Liu et al., 2018; Majka et al., 
bioRxiv preprint doi: https://doi.org/10.1101/2021.11.12.468389; this version posted November 13, 2021. The copyright holder for this preprint (which was not certified by peer review) is the author/funder, who has granted bioRxiv a license to display the preprint in perpetuity. It is made available under aCC-BY-NC-ND 4.0 International license. 2016); (2) RIKEN atlas (Woodward et al., 2018): The atlas is cytoarchitecture based on Nissl-staining contrast. (3) The first Atlas of Marmoset Brain Mapping (MBMv1) (Liu et al., 2018): The borders were delineated based on the highresolution diffusion MRI contrast and parcellated by a structural-connectivitybased approach.

\section{Deep-learning-based individual parcellation generator}

The group-average parcellation described in the preceding sections is desirable for generating parcellations of individual animals. Although applying our grouplevel parcellation to individual animals is feasible as demonstrated in the previous human study (Gordon et al., 2016), we still found misalignments between individuals and cannot be highly consistent with the tendency of the group-average parcellation (MBMv4) when the scanning runs are limited (Fig. 2D-E). Therefore, inspired by previous works (Glasser et al., 2016; Hacker et al., 2013), we trained a multi-layer deep learning network to classify parcels based on the fingerprints from MBMv4. There were two assumptions for this approach: (1) We assumed that individual cortical parcels were close to the group definition after the feature-based surface registration; (2) We assumed that every identified cortical parcel should be in a single class which was the combination of the target parcel and its spatially adjacent parcels (the "searchlight" for the candidate parcel). Thus, the setup of the classifier network was straightforward. Its architecture was as follows (for the graphic reference, see Fig. 4A): for each of the 96 parcels in each hemisphere, a multi-layer deep neural network was designed, which comprised three layers (one input, five hidden, one output) and 384 hidden neurons (a reasonable compromise between accuracy and training speed for the classification). The whole-brain fingerprint of the candidate parcel from the MBMv4 worked as the training set for the network to classify whether or not each vertex in an individual ROI containing the parcel plus all of its neighbor parcels. Because of the spatial overlap of the "searchlight," we excluded the vertices belonging to multiple 
bioRxiv preprint doi: https://doi.org/10.1101/2021.11.12.468389; this version posted November 13, 2021. The copyright holder for this preprint (which was not certified by peer review) is the author/funder, who has granted bioRxiv a license to display the preprint in perpetuity. It is made available under aCC-BY-NC-ND 4.0 International license.

parcels. Then, we applied the same procedure of parcel creation as above, meaning that the borders of each identified parcel became the seeds to expand outward until they met other parcels using the "watershed by flooding" procedure. The whole process of individual parcellation was automatic and implemented using customized Matlab codes (example codes are shared via marmosetbrainmapping.org) combined with MATLAB Deep Learning Network toolbox.

\section{Evaluation by task-activation pattern}

We examined the functional relevance of the borders by evaluating the parcels contained within the fMRI activation pattern to a visual task (Fig. 5A) from our previous study (Tian et al., 2021). A subset of animals from the NIH dataset participated in the visual-choice task, which consisted of watching 20-s-long full-screen movies (visual field is $10 \mathrm{deg} \times$ 8deg) and 16s resting periods (206 trials for marmoset-ID15 and 280 trials for marmoset-ID25). We performed a contrast comparison between the movie-presentation blocks and the resting blocks to generate visual-task activation statistical maps for each session. A mixed-effects analysis was then applied to all statistical maps across sessions by the 3dMEMA command of AFNI to obtain a final statistical map. The map was thresholded at a voxel-wise threshold of $p<0.05$ and a cluster-wise threshold of $p<0.05$ for multiple comparison corrections. To compare the borders consistency of the activation map and parcellation in each hemisphere (for results, see the flat maps in Fig. 5A), we calculated the shortest Euclidean distance between every vertex/voxel in the boundary of the activation map and the vertex/voxel in the boundary of parcels/regions from different parcellations. We considered the parcellation with the shortest overall distances as the best border consistency (for results, see the scatterplots in Fig. 5A).

\section{Evaluation by functional-connectivity gradient spectrum}


bioRxiv preprint doi: https://doi.org/10.1101/2021.11.12.468389; this version posted November 13, 2021. The copyright holder for this preprint (which was not certified by peer review) is the author/funder, who has granted bioRxiv a license to display the preprint in perpetuity. It is made It is widely accepted that the cerebral cortex of multiple species, including both human and macaque primates, is organized along principal functional gradients that provide a spatial framework for the co-existence of multiple large-scale networks operating in a spectrum from unimodal to transmodal functional activity (Margulies et al., 2016; Wang et al., 2019). Therefore, if the MBMv4 parcellation created here accurately represents the functional organization of the marmoset cortex, we can presume that it will also reveal these principal functional gradients. Thus, as in previous studies (Margulies et al., 2016; Vos de Wael et al., 2020), we followed a workflow for gradient identification: we first computed the rs-fMRI functional connectivity (RSFC) based on MBMv4. Next, the RSFC matrix $M_{x, y}$ with the same size as the atlas was made sparse (to a $10 \%$ sparsity), and a similarity matrix $A_{x, y}$ with the normalized angle was computed according to the following equation:

$$
A(x, y)=1-\frac{\cos ^{-1}(\operatorname{cossim}(x, y))}{\pi}
$$

Next, the similarity matrix was decomposed via Laplacian transformation into a set of principal eigenvectors describing the axes of most significant variance using the following equation:

$$
L g=\lambda D g
$$

Where $D_{x, y}=\sum_{y} A(x, y), L$ is the graph Laplacian matrix and the eigenvectors $g$ corresponding to the $m$ smallest eigenvalues $\lambda_{k}$ are used to build the new low-dimensional representation:

$$
\varsigma_{L E}=\left[g_{1}, g_{2}, \ldots, g_{m}\right]
$$


bioRxiv preprint doi: https://doi.org/10.1101/2021.11.12.468389; this version posted November 13, 2021. The copyright holder for this preprint (which was not certified by peer review) is the author/funder, who has granted bioRxiv a license to display the preprint in perpetuity. It is made Finally, the first two axes $g_{1}, g_{2}$ of each parcel were plotted in $2 \mathrm{D}$ space. Meanwhile, we used the scores $g_{1}$ to sort the functional connectivity matrix (for results, see the heatmaps in Fig. 5B).

\section{The whole-brain modeling for the link between structural connectivity and functional connectivity}

As we know, structural connectivity and functional connectivity are closely related to each other. Therefore, lack of structural evidence generally implies biological implausibility for functional connections. Testing whether the cortical parcels created above MBMv4 are accurate representations of the functional areas in the cerebral cortex requires investigation of the underlying structural connectivity. A computational model is a powerful approach to bridge structural and functional connectivity (Breakspear, 2017; Hansen et al., 2015; Honey et al., 2009; Schirner et al., 2018; Wang et al., 2019). In the present study, we implemented a whole-brain model with only two free parameters from previous studies (Deco et al., 2017; Ipina et al., 2020), as outlined below (for a graphic reference, see Fig. 6A):

According to the whole brain parcellations (192 total parcels, 96 per hemisphere from MBMv4 or 464 total brain regions, 232 brain regions per hemisphere from Paxinos Atlas), the structural connectivity between parcels/regions $C_{i, j}$ was estimated from the structural datasets (see examples in Fig. 6C), either DTI data (in-vivo or ex-vivo) or the neuronal tracing data. Then, the local dynamics for every parcel/region $j$ can be properly approximated to the normal form of a Hopf bifurcation:

$$
\frac{d z_{j}}{d t}=\left[a_{j}+i w_{j}\right] z_{j}+z_{j}\left|z_{j}\right|^{2}
$$

In this equation, $z_{j}$ is a complex-valued variable $z_{j}=x_{j}+y_{j}$, and $w_{j}$ is the intrinsic signal frequency of parcel/region $j$, which ranged from $0.04-0.07 \mathrm{~Hz}$ and 
bioRxiv preprint doi: https://doi.org/10.1101/2021.11.12.468389; this version posted November 13, 2021. The copyright holder for this preprint (which was not certified by peer review) is the author/funder, who has granted bioRxiv a license to display the preprint in perpetuity. It is made was determined by the averaged peak frequency of the bandpass-filtered fMRI signals of the parcel/region $j$ (Achard et al., 2006; Buckner et al., 2009; Deco et al., 2017; Glerean et al., 2012; Ponce-Alvarez et al., 2015). $a_{j}$ is a bifurcation free parameter controlling the dynamics of the parcel/region $j$. For $a_{j}<0$, the phase space presents a unique stable and is governed by noise. For $a_{j}>0$, the phase space presents the stable state, giving rise to a self-sustained oscillation. For $a_{j} \approx 0$ the phase presents unstable state, switching back and forth and giving rise to a mixture of oscillation and noise.

The coordinated dynamics of the resting state activity for parcel/region $j$ could be modeled by coupling determined by the above structural connectivity $C_{i, j}$. To ensure the oscillatory dynamics for $a_{j}>0$, the structural connectivity $C_{i, j}$ should be normalized and scaled to 0.2 in a weak coupling condition before simulation starting. The coupled differential equations of the model are the following:

$$
\begin{aligned}
& \frac{d x_{j}}{d t}=\left[a_{j}-x_{j}^{2}-y_{j}^{2}\right] x_{j}-w_{j} y_{j}+G \sum_{i} C_{i, j}\left(x_{i}-x_{j}\right) \beta \eta_{j}(t) \\
& \frac{d y_{j}}{d t}=\left[a_{j}-x_{j}^{2}-y_{j}^{2}\right] y_{j}+w_{j} x_{j}+G \sum_{i} C_{i, j}\left(y_{i}-y_{j}\right) \beta \eta_{j}(t)
\end{aligned}
$$

In this equation, $G$ is another free parameter representing the fixed global coupling factor that scales structural connectivity $C_{i, j} \cdot \eta_{j}$ represents additive Gaussian noise in each parcel/region and is scaled by a factor $\beta$ fixed at 0.04 according to previous studies (Deco et al., 2017). Euler-Maruyama algorithm integrated these equations with a time step of 0.1 seconds to accelerate simulation (Sanz Perl et al., 2021).

The free bifurcation parameter $a_{j}$ for parcel/region $j$ could be locally optimized based on fitting the spectral information of the empirical BOLD signals. To 
bioRxiv preprint doi: https://doi.org/10.1101/2021.11.12.468389; this version posted November 13, 2021. The copyright holder for this preprint (which was not certified by peer review) is the author/funder, who has granted bioRxiv a license to display the preprint in perpetuity. It is made achieve this, we filtered the empirical BOLD data in the $0.04-0.25 \mathrm{~Hz}$ band and calculated the power spectrum $p_{j}(f)$ for each parcel $j$ as below:

$$
p_{j}=\frac{\int_{0.04}^{0.07} p_{j}(f) d f}{\int_{0.04}^{0.25} p_{j}(f) d f}
$$

and updated the local bifurcation parameter $a_{j}$ by a gradient descendent strategy:

$$
a_{j}=a_{j}+\eta\left(p_{j}^{\text {empirical }}-p_{j}^{\text {simulated }}\right)
$$

We applied the above optimization process to receive the best bifurcation parameters $a_{j}$ of every parcel/region defined in the parcellations. Once we found the optimized set of bifurcation parameters $a_{j}$, we adjusted the free parameter $G$ within the range of $0-8$ in steps of 0.1 according to a reasonable compromise from previous studies (Deco et al., 2017; Sanz Perl et al., 2021) to simulate the same number of sessions for each animal and the same number of animals. To compare the performance in different atlases, we just needed to compare fitting (similarity) metrics, Pearson's correlation coefficient between the simulated functional connectivity and the one used for the empirical data, when we fixed the same value of parameter $G$.

Here, we selected the best parameter $G$ from the Paxinos atlas for comparison performance with our MBMv4 (see results in Fig. 6B, examples in Fig. 6D-E). Moreover, we also selected the group-averaged functional connectivity from all individuals as the empirical observable for the ultra-high resolution diffusion MRI and neuronal tracing datasets and the individual functional connectivity for the corresponding in-vivo diffusion MRI. 
bioRxiv preprint doi: https://doi.org/10.1101/2021.11.12.468389; this version posted November 13, 2021. The copyright holder for this preprint (which was not certified by peer review) is the author/funder, who has granted bioRxiv a license to display the preprint in perpetuity. It is made References available under aCC-BY-NC-ND 4.0 International license.

Achard, S., Salvador, R., Whitcher, B., Suckling, J., and Bullmore, E. (2006). A

resilient, low-frequency, small-world human brain functional network with highly connected association cortical hubs. J Neurosci 26, 63-72.

10.1523/JNEUROSCI.3874-05.2006.

Breakspear, M. (2017). Dynamic models of large-scale brain activity. Nat Neurosci 20, 340-352. 10.1038/nn.4497.

Buckner, R.L., Sepulcre, J., Talukdar, T., Krienen, F.M., Liu, H., Hedden, T., Andrews-Hanna, J.R., Sperling, R.A., and Johnson, K.A. (2009). Cortical hubs revealed by intrinsic functional connectivity: mapping, assessment of stability, and relation to Alzheimer's disease. J Neurosci 29, 1860-1873.

10.1523/JNEUROSCI.5062-08.2009.

Caruyer, E., Lenglet, C., Sapiro, G., and Deriche, R. (2013). Design of multishell sampling schemes with uniform coverage in diffusion MRI. Magn Reson Med 69, 1534-1540. 10.1002/mrm.24736.

Cox, R.W. (2012). AFNI: what a long strange trip it's been. Neuroimage 62, 743747. 10.1016/j.neuroimage.2011.08.056.

Deco, G., Kringelbach, M.L., Jirsa, V.K., and Ritter, P. (2017). The dynamics of resting fluctuations in the brain: metastability and its dynamical cortical core. Sci Rep 7, 3095. 10.1038/s41598-017-03073-5.

Glasser, M.F., Coalson, T.S., Robinson, E.C., Hacker, C.D., Harwell, J., Yacoub, E., Ugurbil, K., Andersson, J., Beckmann, C.F., Jenkinson, M., et al. (2016). A multi- 
bioRxiv preprint doi: https://doi.org/10.1101/2021.11.12.468389; this version posted November 13, 2021. The copyright holder for this preprint (which was not certified by peer review) is the author/funder, who has granted bioRxiv a license to display the preprint in perpetuity. It is made available under aCC-BY-NC-ND 4.0 International license.

modal parcellation of human cerebral cortex. Nature 536, 171-178.

10.1038/nature18933.

Glerean, E., Salmi, J., Lahnakoski, J.M., Jaaskelainen, I.P., and Sams, M. (2012).

Functional magnetic resonance imaging phase synchronization as a measure of dynamic functional connectivity. Brain Connect 2, 91-101.

10.1089/brain.2011.0068.

Gordon, E.M., Laumann, T.O., Adeyemo, B., Huckins, J.F., Kelley, W.M., and Petersen, S.E. (2016). Generation and Evaluation of a Cortical Area Parcellation from Resting-State Correlations. Cereb Cortex 26, 288-303.

10.1093/cercor/bhu239.

Hacker, C.D., Laumann, T.O., Szrama, N.P., Baldassarre, A., Snyder, A.Z., Leuthardt, E.C., and Corbetta, M. (2013). Resting state network estimation in individual subjects. Neuroimage 82, 616-633.

10.1016/j.neuroimage.2013.05.108.

Hansen, E.C., Battaglia, D., Spiegler, A., Deco, G., and Jirsa, V.K. (2015). Functional connectivity dynamics: modeling the switching behavior of the resting state.

Neuroimage 105, 525-535. 10.1016/j.neuroimage.2014.11.001.

Honey, C.J., Sporns, O., Cammoun, L., Gigandet, X., Thiran, J.P., Meuli, R., and Hagmann, P. (2009). Predicting human resting-state functional connectivity from structural connectivity. Proc Natl Acad Sci U S A 106, 2035-2040.

10.1073/pnas.0811168106. 
bioRxiv preprint doi: https://doi.org/10.1101/2021.11.12.468389; this version posted November 13, 2021. The copyright holder for this preprint (which was not certified by peer review) is the author/funder, who has granted bioRxiv a license to display the preprint in perpetuity. It is made available under aCC-BY-NC-ND 4.0 International license.

Ipina, I.P., Kehoe, P.D., Kringelbach, M., Laufs, H., Ibanez, A., Deco, G., Perl, Y.S.,

and Tagliazucchi, E. (2020). Modeling regional changes in dynamic stability during sleep and wakefulness. Neuroimage 215, 116833.

10.1016/j.neuroimage.2020.116833.

Jenkinson, M., Beckmann, C.F., Behrens, T.E., Woolrich, M.W., and Smith, S.M. (2012). Fsl. Neuroimage 62, 782-790. 10.1016/j.neuroimage.2011.09.015.

King, M., Hernandez-Castillo, C.R., Poldrack, R.A., Ivry, R.B., and Diedrichsen, J. (2019). Functional boundaries in the human cerebellum revealed by a multidomain task battery. Nat Neurosci 22, 1371-1378. 10.1038/s41593-019-0436-x. Liu, C., Ye, F.Q., Yen, C.C., Newman, J.D., Glen, D., Leopold, D.A., and Silva, A.C. (2018). A digital 3D atlas of the marmoset brain based on multi-modal MRI.

Neuroimage 169, 106-116. 10.1016/j.neuroimage.2017.12.004.

Liu, C., Yen, C.C., Szczupak, D., Tian, X., Glen, D., and Silva, A.C. (2021). Marmoset Brain Mapping V3: Population multi-modal standard volumetric and surfacebased templates. Neuroimage 226, 117620. 10.1016/j.neuroimage.2020.117620. Liu, C.R., Ye, F.Q., Newman, J.D., Szczupak, D., Tian, X.G., Yen, C.C.C., Majka, P., Glen, D., Rosa, M.G.P., Leopold, D.A., and Silva, A.C. (2020). A resource for the detailed 3D mapping of white matter pathways in the marmoset brain. Nature Neuroscience 23, 271-+. 10.1038/s41593-019-0575-0.

Majka, P., Bednarek, S., Chan, J.M., Jermakow, N., Liu, C., Saworska, G., Worthy, K.H., Silva, A.C., Wojcik, D.K., and Rosa, M.G.P. (2021). Histology-Based Average Template of the Marmoset Cortex With Probabilistic Localization of 
bioRxiv preprint doi: https://doi.org/10.1101/2021.11.12.468389; this version posted November 13, 2021. The copyright holder for this preprint (which was not certified by peer review) is the author/funder, who has granted bioRxiv a license to display the preprint in perpetuity. It is made available under aCC-BY-NC-ND 4.0 International license.

Cytoarchitectural Areas. Neuroimage 226, 117625.

10.1016/j.neuroimage.2020.117625.

Majka, P., Chaplin, T.A., Yu, H.H., Tolpygo, A., Mitra, P.P., Wojcik, D.K., and Rosa, M.G. (2016). Towards a comprehensive atlas of cortical connections in a primate brain: Mapping tracer injection studies of the common marmoset into a reference digital template. J Comp Neurol 524, 2161-2181. 10.1002/cne.24023. Marcus, D.S., Harwell, J., Olsen, T., Hodge, M., Glasser, M.F., Prior, F., Jenkinson, M., Laumann, T., Curtiss, S.W., and Van Essen, D.C. (2011). Informatics and data mining tools and strategies for the human connectome project. Front Neuroinform 5, 4. 10.3389/fninf.2011.00004.

Margulies, D.S., Ghosh, S.S., Goulas, A., Falkiewicz, M., Huntenburg, J.M., Langs, G., Bezgin, G., Eickhoff, S.B., Castellanos, F.X., Petrides, M., et al. (2016). Situating the default-mode network along a principal gradient of macroscale cortical organization. Proc Natl Acad Sci U S A 113, 12574-12579.

10.1073/pnas.1608282113.

Papoti, D., Yen, C.C., Hung, C.C., Ciuchta, J., Leopold, D.A., and Silva, A.C. (2017).

Design and implementation of embedded 8-channel receive-only arrays for whole-brain MRI and fMRI of conscious awake marmosets. Magn Reson Med 78, 387-398. 10.1002/mrm.26339.

Paxinos, G., Watson, C., Petrides, M., Rosa, M., and Tokuno, H. (2012). The marmoset brain in stereotaxic coordinates (Elsevier Academic Press). 
bioRxiv preprint doi: https://doi.org/10.1101/2021.11.12.468389; this version posted November 13, 2021. The copyright holder for this preprint (which was not certified by peer review) is the author/funder, who has granted bioRxiv a license to display the preprint in perpetuity. It is made available under aCC-BY-NC-ND 4.0 International license.

Pierpaoli, C. (2010). Quantitative brain MRI. Top Magn Reson Imaging 21, 63.

10.1097/RMR.0b013e31821e56f8.

Ponce-Alvarez, A., Deco, G., Hagmann, P., Romani, G.L., Mantini, D., and

Corbetta, M. (2015). Resting-state temporal synchronization networks emerge

from connectivity topology and heterogeneity. PLoS Comput Biol 11, e1004100.

10.1371/journal.pcbi.1004100.

Sanz Perl, Y., Pallavicini, C., Perez Ipina, I., Demertzi, A., Bonhomme, V., Martial,

C., Panda, R., Annen, J., Ibanez, A., Kringelbach, M., et al. (2021). Perturbations in dynamical models of whole-brain activity dissociate between the level and stability of consciousness. PLoS Comput Biol 17, e1009139.

10.1371/journal.pcbi.1009139.

Schirner, M., McIntosh, A.R., Jirsa, V., Deco, G., and Ritter, P. (2018). Inferring multi-scale neural mechanisms with brain network modelling. Elife 7.

10.7554/eLife.28927.

Silva, A.C., Liu, J.V., Hirano, Y., Leoni, R.F., Merkle, H., Mackel, J.B., Zhang, X.F., Nascimento, G.C., and Stefanovic, B. (2011). Longitudinal functional magnetic resonance imaging in animal models. Methods Mol Biol 711, 281-302.

10.1007/978-1-61737-992-5_14.

Tian, X., Silva, A.C., and Liu, C. (2021). The Brain Circuits and Dynamics of Curiosity-Driven Behavior in Naturally Curious Marmosets. Cereb Cortex 31, 4220-4232. 10.1093/cercor/bhab080. 
bioRxiv preprint doi: https://doi.org/10.1101/2021.11.12.468389; this version posted November 13, 2021. The copyright holder for this preprint (which was not certified by peer review) is the author/funder, who has granted bioRxiv a license to display the preprint in perpetuity. It is made Tustison, N., Avants, B., Cook, P., Kim, J., Whyte, J., Gee, J., Ahlers, S., and Stone,

J. (2011). Multivariate Analysis of Diffusion Tensor Imaging and Cortical

Thickness Maps in a Traumatic Brain Injury (Tbi) Cohort Using Advanced

Normalization Tools (Ants). J Neurotraum 28, A111-A111.

Vos de Wael, R., Benkarim, O., Paquola, C., Lariviere, S., Royer, J., Tavakol, S., Xu,

T., Hong, S.J., Langs, G., Valk, S., et al. (2020). BrainSpace: a toolbox for the

analysis of macroscale gradients in neuroimaging and connectomics datasets.

Commun Biol 3, 103. 10.1038/s42003-020-0794-7.

Wang, P., Kong, R., Kong, X., Liegeois, R., Orban, C., Deco, G., van den Heuvel, M.P., and Thomas Yeo, B.T. (2019). Inversion of a large-scale circuit model reveals a cortical hierarchy in the dynamic resting human brain. Sci Adv 5, eaat7854. 10.1126/sciadv.aat7854.

Woodward, A., Hashikawa, T., Maeda, M., Kaneko, T., Hikishima, K., Iriki, A., Okano, H., and Yamaguchi, Y. (2018). The Brain/MINDS 3D digital marmoset brain atlas. Sci Data 5, 180009. 10.1038/sdata.2018.9. 


\section{Supplementary Information}

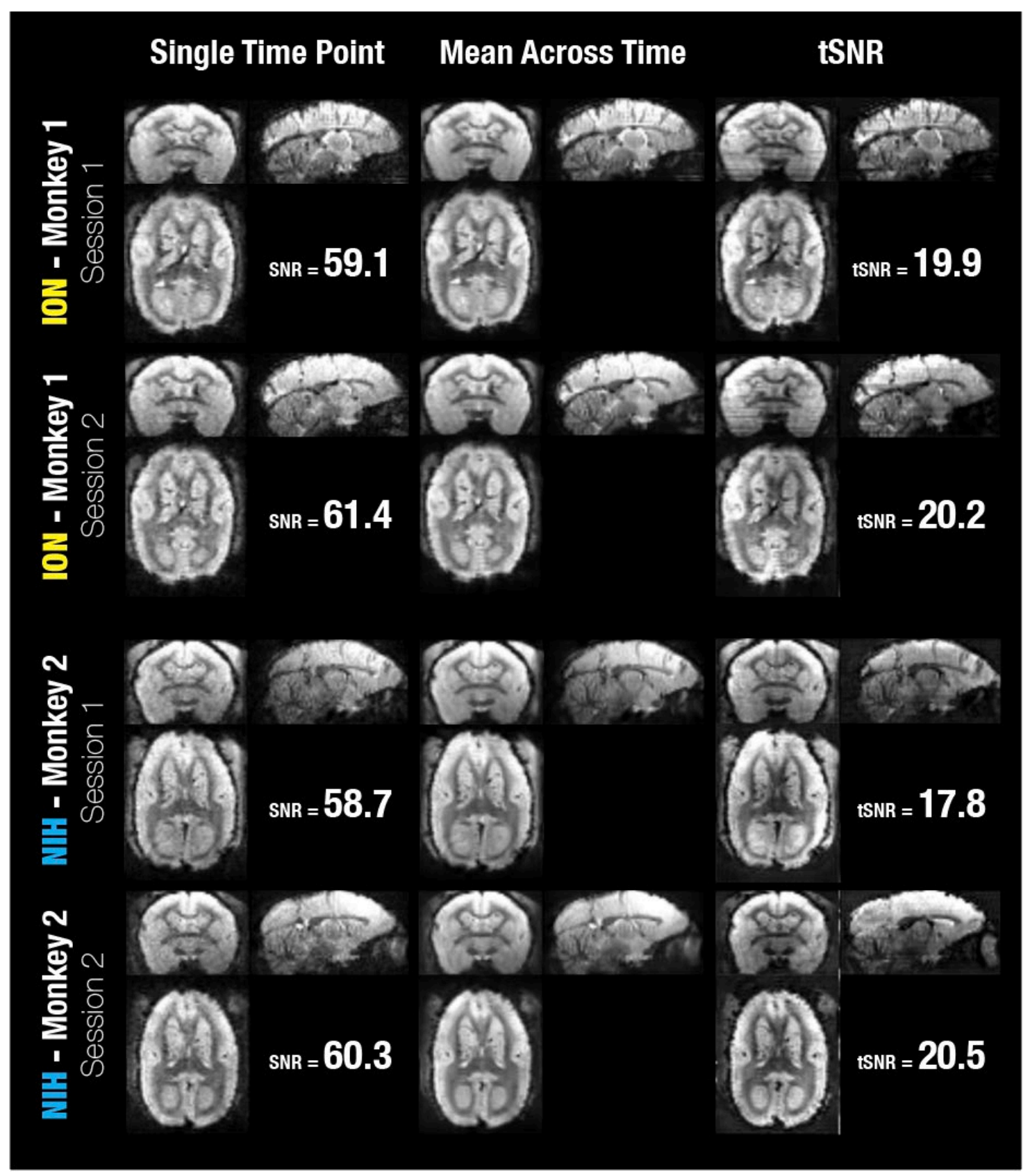

Figure S1. Similar data quality of the ION and the NIH dataset. Single time points, mean images (averaged across time for one fMRI run), and tSNR images (calculated from one fMRI run) are presented for four sessions of two flagship monkeys from ION and NIH, respectively. 


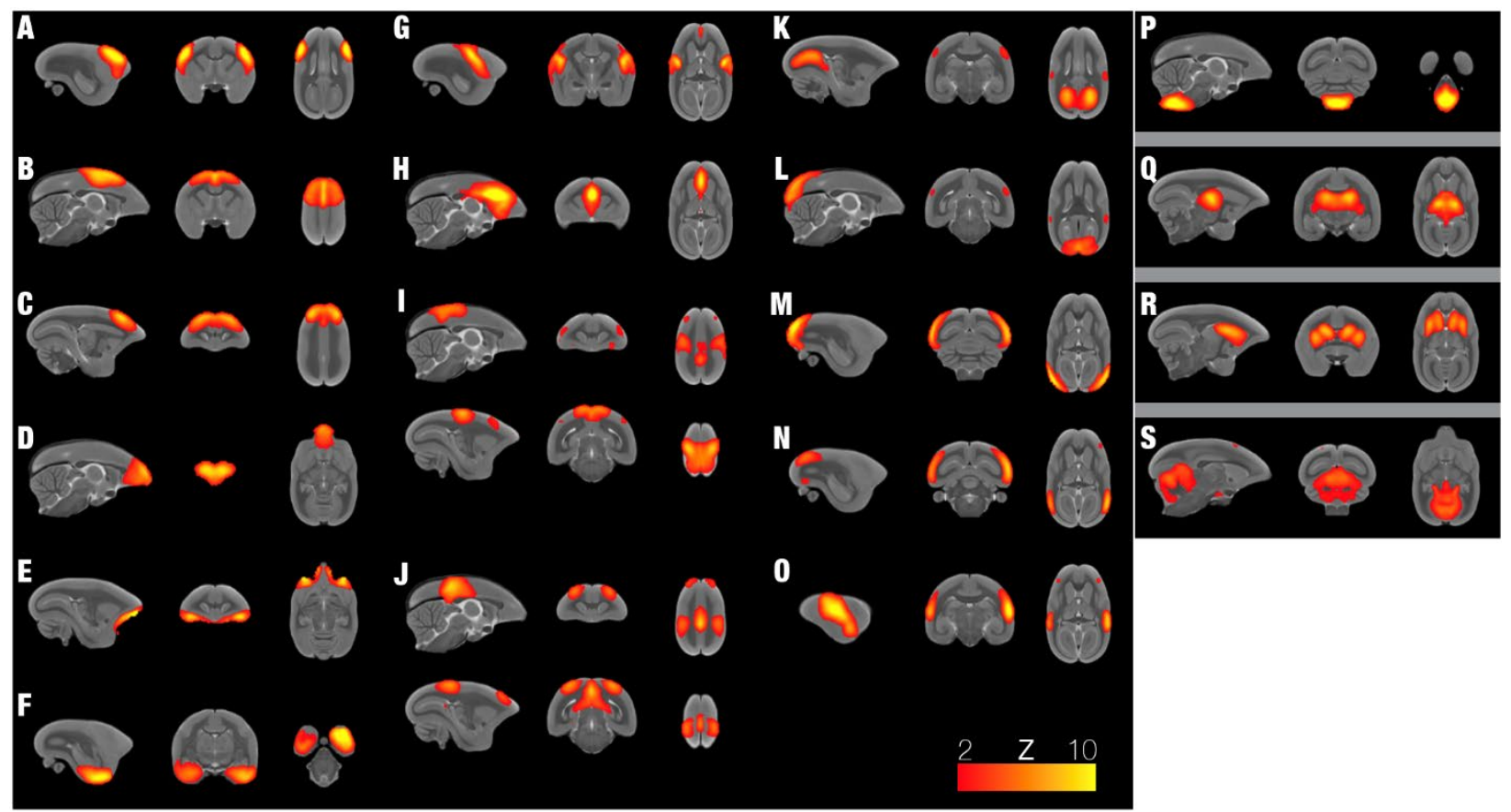

Figure S2. Complete identified resting-state functional networks (in Volume show).

We found 19 networks by group-ICA analysis, including $(A)$ the ventral somatomotor, $(B)$ the dorsal somatomotor, $(C)$ the premotor, $(D)$ the frontal pole, $(E)$ the orbital frontal cortex, (F) the parahippocampus/temporal pole, (G-H) the salience-related network, (I-J) two trans-modal networks, which are frontoparietal (I) and the default-network-related (J), the visual-related networks from primary visual cortex (K-M) to functional higher-level regions $(N-O)$ and subcortical networks, the brain stem $(P)$, the thalamus $(Q)$, the striatum $(R)$, and the cerebellum (S). 


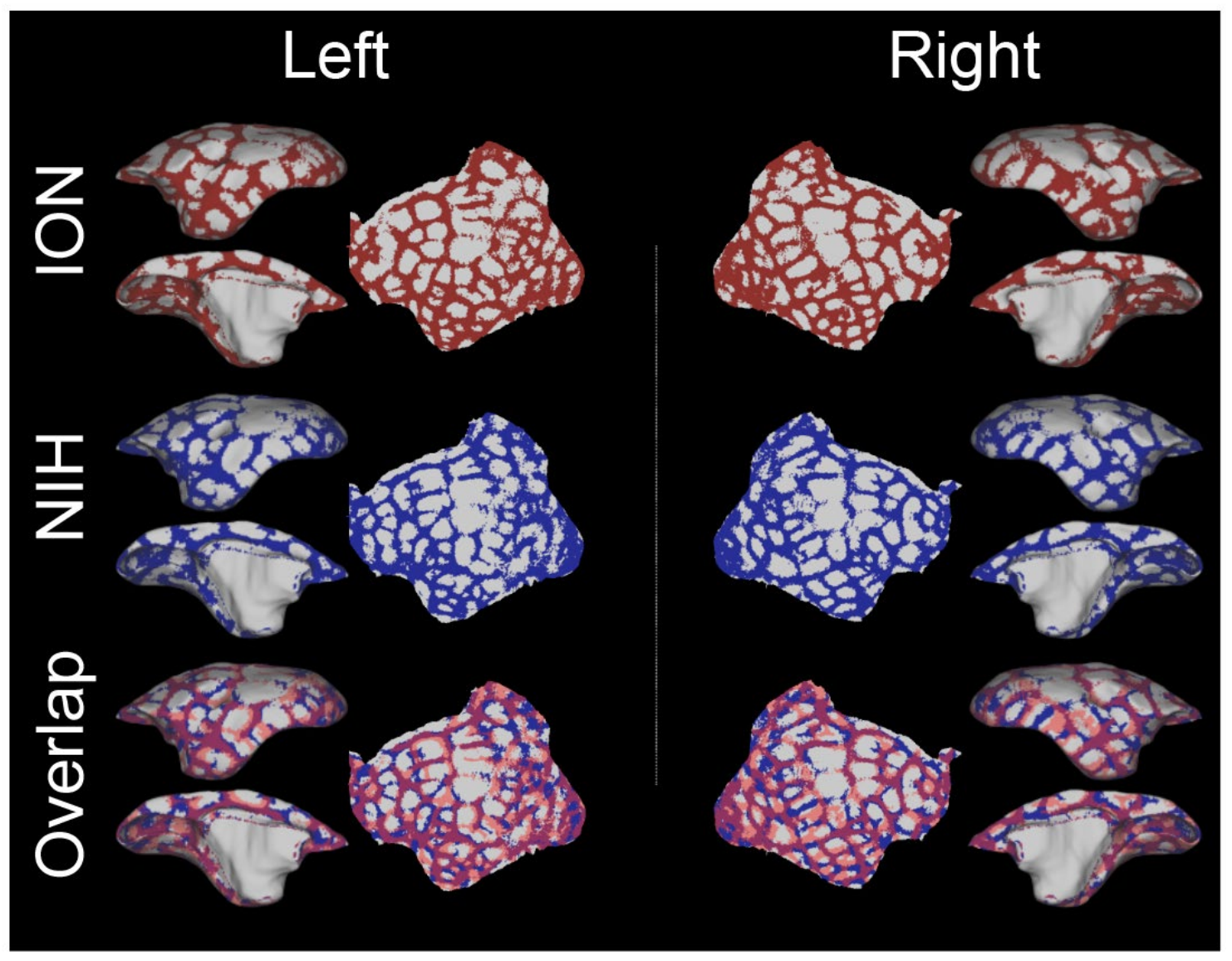

Figure S3. Boundary maps in both hemispheres from NIH and ION Dataset are highly similar. Top and middle panel: The boundary maps in both hemispheres from $\mathrm{NIH}$ and ION datasets after thresholding both at the 75th percentile of boundary map values. Bottom: The comparison between two boundary maps (Top and middle) in both hemispheres. Light blue: NIH boundaries; pink: ION boundaries; purple: the overlapping boundaries between datasets. 

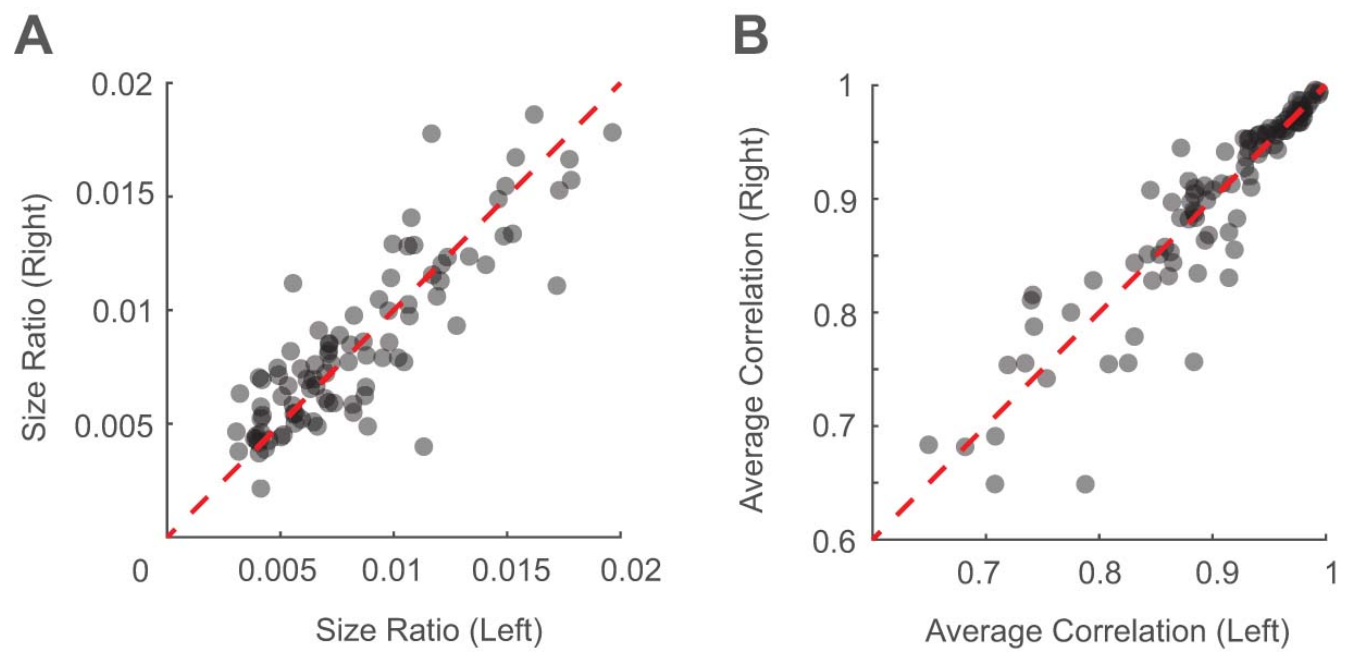

Figure S4. The functional parcels are highly similar across the hemisphere. (A) The parcel sizes. (B) The functional connectivity patterns of parcels. The dashed line represents the diagonal line. 


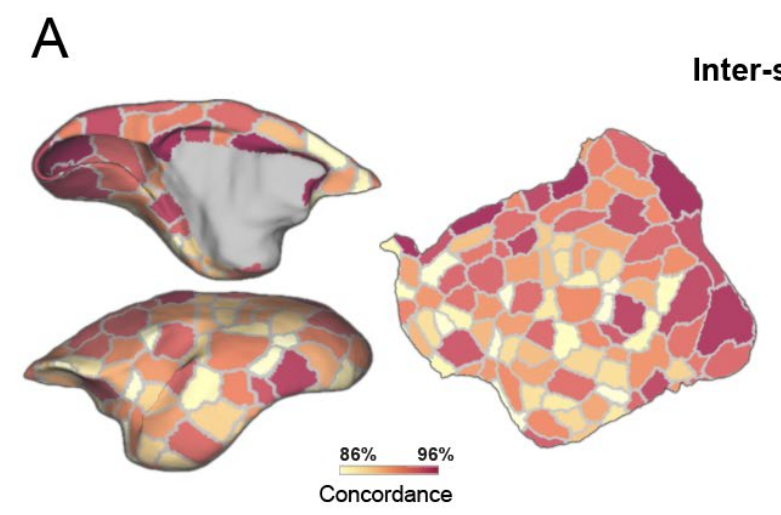

nter-subject

B

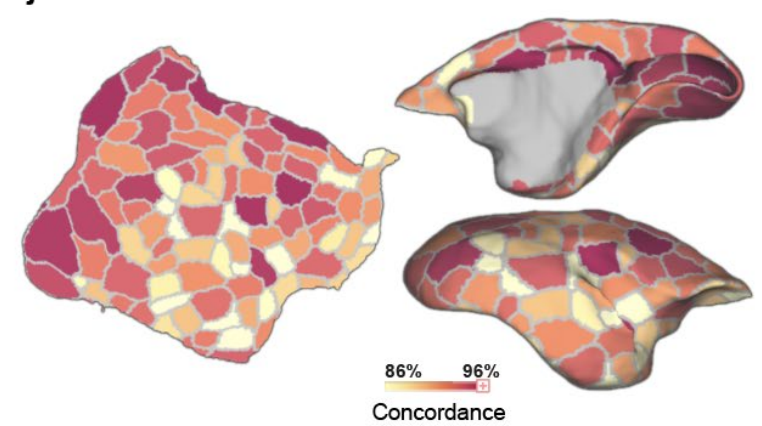

Inter-session
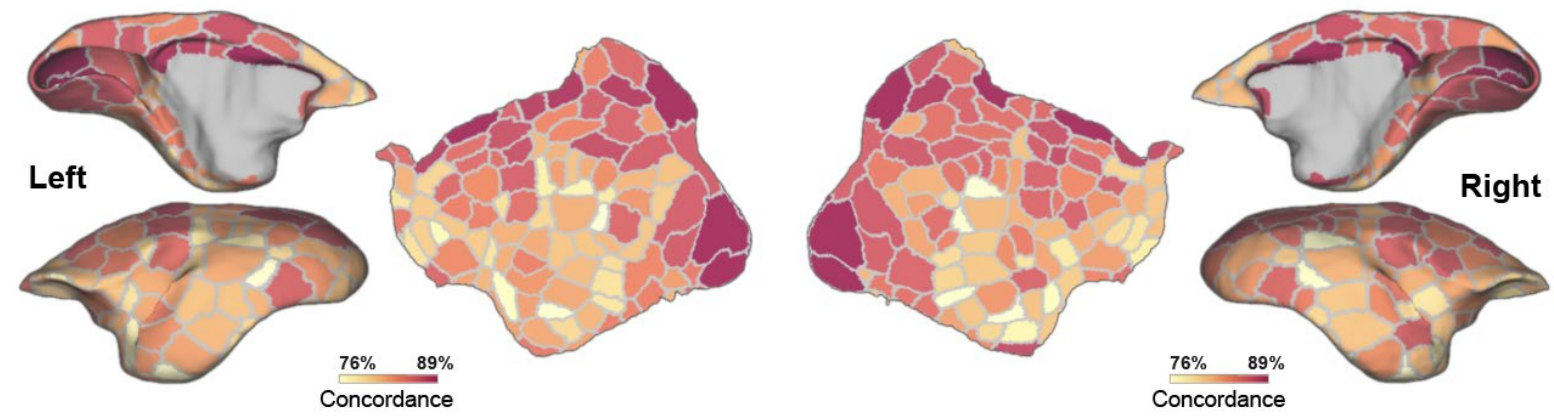

Figure S5. The variation of individual mapping parcels by the deep neural network. (A) The concordance of inter-subject parcels. (B) The concordance of inter-session parcels. 
bioRxiv preprint doi: https://doi.org/10.1101/2021.11.12.468389; this version posted November 13,2021 . The copyright holder for this preprint (which was not certified by peer review) is the author/funder, who has granted bioRxiv a license to display the preprint in perpetuity. It is made available under aCC-BY-NC-ND 4.0 International license.

Table S1. The additional details of datasets. The first and second sheets are the detailed information of recruited marmosets from ION and $\mathrm{NIH}$, respectively. 